\title{
The Emperor's New Metaphysics of Powers
}

\author{
Stephen Barker \\ University of Nottingham \\ stephen.barker@nottingham.ac.uk
}

\begin{abstract}
This paper argues that the new metaphysics of powers, also known as dispositional essentialism or causal structuralism, is an illusory metaphysics. I argue for this in the following way. I begin by distinguishing three fundamental ways of one might see facts of physical modality - facts about physical necessitation and possibility, causation, disposition, and chanceas being grounded in the world. The first way, call it the $1^{\text {st }}$ degree, is that the actual world, or all worlds, in their entirety, are the source of physical modality. Humeanism is the best known such approach, but there are other less well-known approaches. The second way, the $2^{\text {nd }}$ degree, is that the source of physical modality lies in certain $2^{\text {nd }}$-order facts, involving a relation between universals. Armstrong's necessitarianism and other views are $2^{\text {nd }}$-degree. The third way, the $3^{\text {rd }}$ degree, holds that properties themselves are the source of physical modality. This is the powers view. I examine four ways of developing the $3^{\text {rd }}$ degree: relational constitution, graph-theoretic structuralism, dispositional roles, and powerful qualities. All these ways are either incoherent, or just disguised versions of the $1^{\text {st }}$-degree. The new metaphysics of powers is illusory. With the collapse of the $3^{\text {rd }}$ degree, the $2^{\text {nd }}$ degree, the necessitarian view of law, collapses as well. I end the paper with some reflections on the $1^{\text {st }}$ degree, on the problem of explaining necessary connections between distinct existences, and on the dim prospects of holist ontology.
\end{abstract}

\section{Introduction: Three Degrees of Physical Modal Involvement}

There is much talk in the land of a new metaphysics of properties that transforms our understanding of physical modality, that is, of physical necessitation and possibility, causation, disposition, and chance. The heralded new metaphysics is the powers view, known often as dispositional essentialism. ${ }^{1}$ Superficially, the powers view seems to offer a new metaphysics of reality. I shall argue, however, that this is an illusion. The powers view is at best a notational variant on other views, which, when properly understood, are evidently not powers views. The illusion of a new metaphysics is generated by a failure to articulate crucial ideas to do with quidditism, necessary connection, relational constitution, and the distinction between essential and necessary properties. To show this, I introduce a taxonomy for classifying theories of physical modality, which turns on three degrees of involvement that physical modality might have in relation to reality at large.

\footnotetext{
${ }^{1}$ Some proponents are: Shoemaker (1980), Ellis and Lierse (1994), Ellis (2001), Molnar (2003), Heil (2003), Mumford (2004), Bird (2006, 2007), Martin (2008), Whittle (2008, 2009), Jacobs (2011), Mumford and Anjum (2011), and Tugby (2012). Some call the view causal structuralism-see Hawthorne 2001.
} 
The $1^{\text {st }}$ degree is that facts of physical modality are determined by, or supervene upon, features of the world or worlds in totality. The best-known $1^{\text {st }}$-degree view is Humeanism, whose chief modern proponent is Lewis (1986). For Lewis the actual world is a vast mosaic of particular matters of fact, that is, instantiations of properties at particular regions across a four-dimensional spacetime continuum. ${ }^{2}$ Laws are regularities in this mosaic that meet certain conditions, viz, the statements expressing these regularities are members of a best system describing the actual world.

Because, on the Lewisian view, facts of physical modality are determined by prior patterns of property distribution, the identity of properties has to be fixed by something prior to facts of physical modality. Properties are quiddities in Black's (2000) sense. A property is a quiddity if and only if what fixes the identity of the property is qualitative, in the sense that it is a suchness, a way things might be, that is either structureless (primitive) or structured but in a way that does not involve modal role. In short, a property is quidditistic if and only if what determines the property's identity across possible worlds is not the causal-nomological roles into which the property may enter. ${ }^{3}$

The $2^{\text {nd }}$ degree of physical modal involvement is that facts of physical modality are not metaphysically determined by features of worlds as a whole, such as patterns in the mosaic of fact, but are determined at a more fine-grained level. On the $2^{\text {nd }}$-degree view, what does the fixing is, primarily, a $2^{\text {nd }}$-order relation holding between natural properties. The best-known version of the $2^{\text {nd }}$ degree is the view provided by Armstrong (1983), Dretske (1977), and Tooley (1977). I will just call this the Armstrong view here. According to the Armstrong view, a law involving two natural properties, $F$ and $G$ is a relational fact of the form $N[F, G]$, where $N$ is a nomological relation. $N$ is a $2^{\text {nd }}$-order relation whose instantiation by $F$ and $G$ is meant to determine the patterns of causation and physical necessitation involving events into which $F$ and $G$ enter. So if $N[F, G]$ holds at a world $w$, then, as a result, whenever an object at $w$ has $F$, it has $G$. The Armstrong view embraces quidditism about

\footnotetext{
2 By an instantiation of a property I mean the instancing of a property when a thing possesses it. So if redness is possessed by this apple, there is an instancing of redness at the spatiotemporal region where the apple is. Often I leave out reference to objects and say that instantiation of one property is linked to another.

${ }^{3}$ A quiddity could also called a categorical or qualitative property.
} 
properties, since natural properties are not constituted (fully or partially) by their entering $N$ relations. What fixes their identity is a nature lying behind their modal roles, which may simply be a primitive suchness or a non-modal structure. In short, it is a quiddity.

Lewis's Humeanism is the best known $1^{\text {st }}$-degree view, and the Armstrongian approach the best known $2^{\text {nd }}$ degree approach. But there are other $1^{\text {st }}$ degree and $2^{\text {nd }}$ degree views out there, which differ significantly from each other, though all of them embrace quidditism. ${ }^{4}$ We shall meet these other $1^{\text {st }}-$ and $2^{\text {nd }}$-degree views below.

Finally, there is the $3^{\text {rd }}$ degree of physical modal involvement. This is where powers ontology is meant to be. For the $3^{\text {rd }}$ degreer, properties are inherently modally potent or active; properties are, by their very natures, internally connected to physical modality. Whereas $2^{\text {nd }}$-degree theorists say that it is nomologial facts of the form $N[F, G]$ that metaphysically determine the patterns of property-instantiation, $3^{\text {rd }}$ degreers say that it is the properties themselves that metaphysically determine the patterns of property-instantiation into which these properties enter. So $F$ 's instantiation will, in and of itself, require, for example, that other properties $G$ are instantiated, or have a tendency to be instantiated, and so on. That is the inherent potency of properties in action. What $3^{\text {rd }}$-degree theorists purport to offer then is a world of properties in which there is no split between modally inert quiddities, on the one hand, and physical modality, as a kind of order imposed upon passive beings, on the other. Intuitively, this looks right. Physics seems to give us a picture of basic properties in which property natures are purely dispositional, exhausted by causal role. Properties are defined by what they $d o$. We should then shun any quidditistic, property substratum, beneath the physical roles, since it looks like an artefact of bad metaphysics.

It is one thing to announce a metaphysical program. It is another thing to carry it out. Until we have a $3^{\text {rd }}$-degree metaphysics on paper, we do not know if the intuition that modality flows from properties isn't just an illusion we have about the world. Below I argue that there is no coherent, $3^{\text {rd }}$-degree metaphysics. The crucial issue for the powers view is how modal role is built into, or produced by, the very nature of properties. I look at the four

\footnotetext{
${ }^{4}$ Both the $1^{\text {st }}$-degree and $2^{\text {nd }}$-degree conceptions need some development to give accounts of physical modality in indeterministic worlds. I shall not consider these issues in detail here.
} 
main ways this has been conceived: relational constitution, constitution through graphs, functional roles, and qualities grounding powers. I argue that these approaches, when unpacked, fail to deliver any powers ontology. They are either incoherent or simply variants of $1^{\text {st }}$-degree theories. ${ }^{5}$ Moreover, I show that if the $3^{\text {rd }}$ degree collapses, so does the $2^{\text {nd }}$ degree, since the $2^{\text {nd }}$ degree's conception of a $2^{\text {nd }}$-order relation $N$ as a source of necessitation in the world depends on the idea of $N$ being essentially modal. (The $2^{\text {nd }}$ degree is just a kind of $2^{\text {nd }}$-order powers view.) So the only approach left is the $1^{\text {st }}$ degree. That means, as I show below, accepting quidditism and one of the following: Humeanism, brute necessary connections between distinct existences, or brute relations of comparative similarity between worlds. These are not very attractive views, in my eyes, but they are the only ones that remain undefeated by the considerations raised in this paper.

I proceed by setting up my taxonomy of theories of physical modality, $1^{\text {st }}$ - and $2^{\text {nd }}$ degree views. ${ }^{6}$ I then describe and access the four ways to the $3^{\text {rd }}$ degree.

\section{Necessary Connections, Quidditism, and World-Making}

My description of $1^{\text {st }}-, 2^{\text {nd }}, 3^{\text {rd }}$-degree theories of physical modality depends on clarifying a principle that will loom large over much that follows: the thesis that there are no necessary connections between distinct existences. I neither condone nor condemn the principle here. To clarify this principle we need to explicate necessary connection, distinct existence, real possibility, and quidditism.

A necessary connection holds between two things $A$ and $B$ if and only if for every metaphysically possible world, if $A$ exists, then $B$ exists. For example, there is a necessary connection in this sense between a person and their origin, assuming necessity of origin. Or consider two properties $F$ and $G$. Suppose the properties are linked in this way: in every possible world in which $O$ instantiates $F$, then $O$ instantiates $G$. For example, in every world

\footnotetext{
${ }^{5}$ My attack on the $3^{\text {rd }}$ degree encompasses global theories - or pandispositionalist-(Mumford 2005, Bird 2007), partial theories, according to which some properties are qualitative (Ellis 2001, Molnar 2003), and theories that claim that properties are somehow, powerful qualities (Heil 2003, Martin 2008).

${ }^{6}$ Another approach to physical modality is to opt out of metaphysics and treat laws as primitive but not open to metaphysical analysis. See, for example, Carroll 1994. I won't consider this kind of approach here.
} 
in which $O$ is scarlet, $O$ is red. The state, $O$ is scarlet has a necessary connection to $O$ is red, since if the first exists, the second must. And consequently, in a secondary sense, the properties, scarlet and red have a necessary connection. ${ }^{7}$

Consider the term distinct existences. The term is a rather slippery one from the point of view of our concerns here. It can mean not identical. But the principle, no necessary connections between non-identical things is clearly false. For example, a mereological whole $W$ is non-identical to a proper part $O$, but $W$ 's existence necessitates $O$. The thesis we are after concerns another relation. Say that $A$ contains $B$ if and only if $A$ ontologically depends on $B$. In other words, in a real definition of $A$ - an account of what the inherent nature of $A$ is - there is reference to the inherent nature of $B$. For example, the real definition of mereological whole $W$ involves reference to $O$. So, $W$ contains $O$. Or, a real definition of what being hand $h$ consists in might make reference to the body $b$ it is part of: $h$ ontologically depends on $b$. That means, in our technical sense, $h$ contains $b$. On the other hand, the real definition of a grain of sand $g$ does not involve reference to the pile $p$ it may be part of. So, $g$ does not contain $p$. Or it might be that the real definition of what the body $b$ is does not make reference to its hand, $h$, so $b$ does not contain $h$.

Here then is a formulation of the principle that there are no necessary connections between distinct existences, $N N C$ below, to which we refer in this paper: ${ }^{8}$

$N N C$ : If $A$ does not contain $B$ then it is metaphysically possible for $A$ to exist without $B$ existing.

$N N C$ seems to be confirmed by many cases. Sand-grain $g$ does not contain pile $p-g$ is not ontologically dependent on $p$-so surely, $g$ can exist without $p$. However, $N \boldsymbol{N C}$ is challenged by many other cases. Take the fact $\boldsymbol{C L}$ :

$\boldsymbol{C L}$ : An object $x$ 's instantiation of scarlet necessitates $x$ 's instantiation of red.

\footnotetext{
${ }^{7}$ Below, I am neutral on questions of modal realism and transworld identity.

8 See Wilson 2010 for other formulations of the principle of no necessary connections between distinct beings. See Lewis 1986 for a characterization of the principle, conceived as one of free modal recombination.
} 
It is plausible to think that a fact, $[O$ is red $]$ is distinct from [ $O$ is scarlet $].{ }^{9}$ The real definition of scarlet (presumably) is not: $x$ is red \& $x$ is $Z$, for some condition $Z$. Nor is it clear that red is the disjunctive property of its determinants. Even if it were, that would not mean $\boldsymbol{C L}$ did not violate $\boldsymbol{N N C}$. If red and scarlet exist-I assume here they do - then $\boldsymbol{C L}$ implies that the fact [ $O$ is scarlet] necessitates a disjunctive one, [O is scarlet $v$ crimson $v$...], etc. But $[O$ is scarlet] does not contain the disjunctive fact. The real definition of scarlet does not contain the disjunctive property. $N N C$ is then violated.

Take a case slightly closer to our concerns, like $\boldsymbol{M}$ below, where $F^{1}$ and $F^{2}$ and $G$ are natural properties - say $F^{1}$ is having a certain mass $m$ and $F^{2}$ is being under some net force $f$, and $G$ is having a certain acceleration $a$ :

M: An object $x$ 's instantiation of $F^{1}$ and $F^{2}$ necessitates $x$ 's instantiation of $G$.

$\boldsymbol{M}$ looks like a violation of $\boldsymbol{N N C}$. That is because the joint state of $O^{\prime}$ 's being $F^{1}$ (having mass $m$ ) and $F^{2}$ (being under force $f$ ) is not constituted, in part, by $O$ 's accelerating. This holds even if the properties involved are dispositional and defined in terms of stimulus and response conditions. Say having $F^{1}$ (mass $m$ ) is the disposition to have acceleration $a$, given force $f$. The real definition of being disposed to accelerate does not involve actually accelerating. Hence, the state, [O is $F^{1}$ and $\left.F^{2}\right]$ does not reside, even partly, in $O$ 's accelerating. This is the case, even if $O$ 's acceleration is instantaneous, given $F^{1}$ and $F^{2}$ are instantiated. $O$ 's acceleration is the result of a necessity that goes beyond the mere existence of [ $O$ is $F^{1}$ and $F^{2}$ ]. It is not the necessity of essence, viz, the necessity of being part of the real definition of [ $O$ is $F^{1}$ and $F^{2}$ ], but some other necessity. So, $M$ violates $N N C$.

If we accept a combinatorial conception of worlds, of the kind favoured by Lewis (1986), violations of $N N C$ are impossible. Very roughly, all combinations of entities consistent with their real definitions must appear somewhere in modal space, since worlds are nothing but combinations of such entities: worlds are derivative ontology from combinations. So, inevitably, there will be worlds where an object has $F^{1}$ and $F^{2}$ (a certain

\footnotetext{
${ }^{9}$ Below I use '[...]' to designate facts in the sense of states of affairs in the world.
} 
mass and force) but does not accelerate. Any sense we have of nomological connection between natural properties needs another explanation. (See Sect. 2 below.)

On the other hand, if we want to accept violations of $\boldsymbol{N} \boldsymbol{N} \boldsymbol{C}$, like $\boldsymbol{C L}$ and $\boldsymbol{M}$, we need a more restricted conception of real possibility, so that the space of possible worlds is smaller than the combinatorialist admits. Here's one way of doing this, which is not a way that either $2^{\text {nd }}$ - or $3^{\text {rd }}$-degree theorists can accept (as we shall see). Call brute-modalism the view that it is a basic fact about worlds that regularities like $\boldsymbol{C} \boldsymbol{L}$ or $\boldsymbol{M}$ obtain. Given brute-modalism, the properties in $\boldsymbol{M}$ and $\boldsymbol{C L}$ are quiddities whose instantiations have necessary connections to other quality-instantiations, which just reflect the primitive nature of possible worlds themselves.

You might be puzzled by the idea that brute-modalism involves quidditism about properties. Surely, given $\boldsymbol{M}$, properties like $F^{1}, F^{2}$ and $G$ are necessarily connected to modal roles, that is, to nomological connections to other properties. In which case, isn't $\boldsymbol{M}$ inconsistent with quidditism about properties? This objection assumes Permute:

Permute: A property is a quiddity iff its modal roles change across possible worlds.

Permute is false. Quidditism about properties isn't simply a commitment to properties changing their modal roles across possible worlds. Rather, quidditism is a thesis about the essence or real definition of properties, that is:

Quid: A property $F$ is quidditistic iff its identity is fixed by features that in themselves are non-modal. Its real definition involves only these features.

Thus, quidditism implies that what fixes the identity of the property is qualitative, in the sense that it is a primitive suchness, or a (non-modal) structural nature. (That fits in with Black's (2000: p. 92) definition of quidditism.) What fixes something's identity is very close to that thing's essence (Fine 1994). The essence of something is not simply what's necessary to that thing. Rather, the essence is that which grounds the identity of something. It is that in virtue of which something is the thing it is. It is what its real definition specifies. 
If brute-modalism is correct, then necessary connections are brute facts about worlds. Each world is just a mosaic of local matters of fact involving qualities. Unlike combinatorialism — which reduces possibility to combination of distinct existences - brutemodalism treats possible world as primitive. This may be unattractive, but there's no contradiction involved. Brute-modalism's availability as a coherent hypothesis shows that a thesis about what's essential to properties — what fixes their identity — cannot merely be captured by facts about what's necessary. Given brute-modalism, the truths expressed in $\boldsymbol{C L}$ and $\boldsymbol{M}$ can only be nominal definitions of properties. They can't be real definitions.

You will reject brute-modalism if you think that the patterns of property instances across worlds should be the result of the nature of properties - the patterns somehow flowing from the natures of properties, constraining possibility. This idea requires that worlds be derivative ontology, so they are the result of the nature of properties and some principle of world-making. For the brute-modalist, worlds are not made, but brute realities.

We can capture this alternative idea of properties entering into the making of worlds through a simple analogy. Think of properties as shaped tiles and worlds as surfaces on which tiles can be placed, where there is a mechanism for placing tiles onto surfaces and getting them to settle flatly without spaces between. Each tessellation of a surface corresponds to a possible world. Each tessellation reflects the nature of tiles, since the tilelaying principle is sensitive to shape. In the property-world making picture, tiles are analogues of properties with qualitative natures. The tile-laying principle is the analogue of the world-making principle. So two aspects of reality enter into the making of worlds, as in:

$$
\begin{aligned}
& \begin{array}{c}
\text { World-making Principle } \\
+
\end{array} \\
& \text { Nature of Properties }
\end{aligned}
$$

\section{Worlds}

There is no denial that properties have qualitative nature. On the contrary, it requires that properties have a qualitative nature or aspect. It is just a denial that this qualitative nature is passive, that is, makes no contribution to the possibilities there are. The contrast should be clear. Brute-modalism claims that $\boldsymbol{M}$ is just a brute fact about the patterns we find in worlds. 
Worlds are fundamental bits of how things are. In contrast, property world-making claims worlds are made and the result of prior natures of properties constraining their making.

As we shall see, powers theorists often talk in terms of properties shaping outcomes. This is how properties participate in the activity of the world. The fourth way to the $3^{\text {rd }}$ degree is very much like this (Sect. 8 below). The question is does this world-making picture really make any sense. What exactly is the nature of the world-making principle? The latter cannot merely be a cluster of fundamental laws, like $\boldsymbol{C} \boldsymbol{L}$ and $\boldsymbol{M}$, linking property-instances across possible worlds. If this is the world-making principle then these laws are just basic. But that is just brute-modalism. Rather, the world-making principle must be something that interacts with features of properties resulting in the property instantiations having certain patterns, like $\boldsymbol{C} \boldsymbol{L}$ or $\boldsymbol{M}$. That requires that properties have features $f^{1}, f^{2}$, etc-tiles shapes in our tessellation model-upon which the world-making principle operates. Let $f^{1}, f^{2}, g$ be the features $\left(2^{\text {nd }}\right.$-order properties) of the natural properties, $F^{1}, F^{2}, G$. The idea then is that $\boldsymbol{M}$ holds because of the operation of the world-making principle on $f^{1}, f^{2}, g$.

However, doubts about this picture now emerge. In our tessellation model, laws must govern how the tile-placing system works: by necessity tiles of one type can only fit with tiles of another. These are not purely geometrical laws, since fitting also reflects facts about, tile impenetrability and inflexibility. The world-making principle must likewise be a lawgoverned system. What's the necessity underpinning its laws? If it is just brute, then $f^{1}, f^{2}, g$, etc, are only externally linked to each other in brute necessary connections. But then $F^{1}, F^{2}$, $G$ are only externally linked to each other. If we suppose that the laws governing $f^{1}, f^{2}, g$, etc are in turn determined by an even higher-level world-making principle, then we enter a regress. Property world-making either ends in a regress of ever higher-level occult propertyfeatures - higher level tiles as it were- or $2^{\text {nd }}$-order brute-modalism. Both pictures are bad.

This illustration shows how difficult it may be to escape from brute necessary connections (and brute-modalism). Powers theorists want to affirm the necessity of modal roles. They want necessity to flow from, or be internal to, the inherent nature of the properties. But here's the problem (and not the only one, as we shall see). Physical necessity cannot simply be reduced to the necessity of essence. The necessity in $\boldsymbol{M}$ violates $\boldsymbol{N N C}$. The 
fact $\left[O\right.$ is $F^{1}$ and $\left.F^{2}\right]$ does not contain $O$ 's accelerating $(G)$ as part of its essence. (That holds, as we saw, even if the property $F^{1}$ (having mass $m$ ) has a dispositional essence.) It is not the necessity of essence driving $\boldsymbol{M}$ but some other kind of necessity. The challenge for the $3^{\text {rd }}$ degree is to explain this necessity, or its relation to properties, without collapsing into brutemodalism. That is what, I shall argue, cannot be done. ${ }^{10}$

\section{The $1^{\text {st }}$ and $2^{\text {nd }}$ Degrees}

We have put a set of concepts on the table: distinct existences, necessary connections, quiddities, and ideas about real possibility. We can now begin to appreciate positions within the three degrees of modal involvement. I begin with $1^{\text {st }}$-degree views of physical modality, which all accept that properties are quiddities in the sense of Quid.

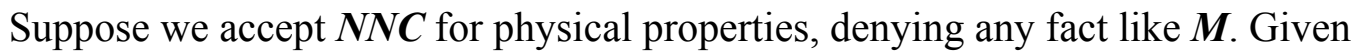
NNC, the pattern of property distribution - the mosaic of events across the spatiotemporal continuum of the actual world - is not metaphysically constrained. We might say that the mosaic is a metaphysical accident. If $\mathbf{N N C}$ is embraced for physical modality, how then can physical laws arise? It is here we meet our first version of the $1^{\text {st }}$ Degree: the Humean conception. The Humean idea is that laws of the actual world are derivative, metaphysically speaking, from the pattern of property instantiations in the actual world. The crudest form of Humeanism is that the regularities are constant conjunctions of $F \mathrm{~s}$ with $G$ s, etc. In the more sophisticated best-system theory, the physical laws correspond to those statements of regularity that appear in a system of general statements that provides the most economical and powerful description of physical reality (Lewis 1986). ${ }^{11}$

\footnotetext{
${ }^{10}$ There is a comparable challenge to explain the necessity behind $\boldsymbol{C L}$ - between the determinant and the determinable. Obviously, we feel intuitively that it is internal in some sense that scarlet goes with red. But cashing out this metaphor of internality with a metaphysical theory is something else.

${ }^{11}$ The Humean combinatorialist position entails wholesale acceptance of $\boldsymbol{N N C}$. But wholesale acceptance of NNC looks unattractive. (See Wilson 2012.) Do we want to deny principles like $\boldsymbol{C} \boldsymbol{L}$ ? A Humean might deny there are any determinables, qua properties, but there are determinable predicates. Thereby they might reduce $\boldsymbol{C} \boldsymbol{L}$ to a definitional truth. I won't consider the virtues of this idea, and related ones here. I note that Humeans can mimic some aspects of the dispositional essentialist picture through a structuralist treatment of processes. See Handfield 2008.
} 
Here's a second $1^{\text {st }}$-degree view. Let us accept the brute-modalist conception of modality sketched above, and propose that natural properties are quiddities that feature in transworld regularities like $\boldsymbol{M}$, which are simply basic facts about possible worlds, and that physical laws are regularities like $\boldsymbol{M}$. Call this view transworld Humeanism. The latter is a $1^{\text {st }}$-degree conception of physical modality since it claims that properties are quiddities and that physical modality is fixed by global features of worlds. Worlds are mosaics of particular matters of quidditistic fact. Regularities like $\boldsymbol{M}$ hold. Why? That is just how it is.

Transworld Humeanism does not have to restrict itself to facts like $\boldsymbol{M}$, linking natural property instantiations to other natural property instantiations. It can also propose necessary connections involving causal processes and propensities, as in:

Necessarily, facts of the form $[x$ is $F]$, etc. cause facts of the form $[y$ is $G]$.

Necessarily, where a fact $[x$ is $F]$, etc. obtains, there is a propensity for $x$ to be $G$. Transworld Humeanism would then have to give some further theory about what causal processes and propensities are. It is not obvious that it could not do that. At the very least, it might treat causation and propensity as primitive. That is not an attractive position, perhaps, but it is consistent with brute-modalism (and transworld Humeanism).

Here's a third $1^{\text {st }}$-degree view: affirm combinatorialism about modality, and thus quidditism about properties, but contend that it is just a primitive fact that certain relations of comparative similarity hold between the actual world and other worlds. These primitive relations of world-comparative similarity would be the grounds for counterfactuals holding at the actual world. The counterfactuals then fix the facts of physical modality. So, for example, the counterfactual, Were O with mass $m$ to have been subjected to force f, it would have had acceleration $a$, holds because of brute facts of similarity between worlds.

In this version of the $1^{\text {st }}$ degree, we are not proposing that the comparative similarity relations are the result of patterns of property distribution in the worlds. That is Lewis's Humean proposal, according to which property-distribution patterns fix the laws, and comparative similarity relations are determined by degrees of law-violation (relative to the laws of the actual world) and divergence from particular matters of fact in the actual worldsee Lewis 1979. Instead, on the present proposal, the comparative similarity relations are 
primitive, and not linked as such to patterns of property distribution across worlds. This naturally enough makes the view relatively unattractive, but there it is.

This primitive world-similarity conception is a $1^{\text {st }}$-degree view since, according to it, irreducible features of worlds as a whole are the basis of physical modality. Moreover, properties are quidditistic. Worlds in themselves are prior to the relations of comparative similarity holding between them. Properties are ontologically prior to the counterfactual dependencies holding at any world. Thus modal roles have no involvement in fixing the identity of properties, and so by Quid, properties are quiddities. This version of the $1^{\text {st }}$ degree does not involve any denial of $N N C$. We are not supposing that the metaphysically possible worlds exclude any combinations of distinct things. It is the primitive comparative similarity relation that does all the work in securing the facts of physical modality, not any necessary connections violating $N N C .12$

\subsection{The $2^{\text {nd }}$ Degree}

That is the $1^{\text {st }}$ degree. Whereas the $1^{\text {st }}$ degreer sees modal facts being fixed by world-sized facts, the $2^{\text {nd }}$ degreer sees modal facts being fixed by certain $2^{\text {nd }}$-order relational facts. The paradigm $2^{\text {nd }}$-degree view is Armstrong's. According to Armstrong, there is a $2^{\text {nd }}$-order relation, $N$, that can be instantiated by natural properties, $F$ and $G$, forming a fact $N[F, G]$. This $2^{\text {nd }}$-order fact, $N[F, G]$ metaphysically determines patterns of instantiation of $F$ and $G$ in events/facts throughout the spatiotemporal continuum, as in:

$\boldsymbol{M M}: N[F, G]$ necessitates that every $x$ that is $F$ is $G .^{13}$

This idea of a necessitating $2^{\text {nd }}$-order fact is the essence of the $2^{\text {nd }}$-degree view.

Note several things: First, $2^{\text {nd }}$ degreers will need a family of $N$-relations. They will require $2^{\text {nd }}$-order relations linking more than two properties, since laws might link more than

\footnotetext{
12 Lange's (2009) theory of laws might be a version of this approach. Roughly, for Lange, laws are regularities that are members of the greatest set of regularities that are invariant under (non-counterlegal) counterfactual suppositions. Counterfactuals, for Lange, cannot depend on laws, since lawhood depends on them. This accords with the idea that counterfactuals depend on irreducible relations of comparative similarity between worlds.

${ }^{13}$ Armstrong calls facts like $N[F, G]$ laws. In physics, laws are functional relations between determinables like force, mass, and acceleration, expressed by equalities like $f=m a$. Functional laws can be thought of as grounded by the network of $N$-relations between natural properties, the determinants of these determinables (physical quantities). That means functional laws supervene on Armstrong's necessitations.
} 
two properties. So, for $2^{\text {nd }}$ degreers, a law linking $F^{1}$ (having mass $m$ ), $F^{2}$ (being under force $f$ ) and $G$ (having acceleration $m$ ) has the form: $N\left[\left(F^{1}, F^{2}\right), G\right]$. (In what follows I use $N[F, G]$ as emblematic for all these cases.) Secondly, just as the transworld Humean can couch their position in terms of causal processes and propensities, $2^{\text {nd }}$-degree theorists can do the same. So, for example, $2^{\text {nd }}$ degreers might hold that there is a $2^{\text {nd }}$-order relation, $T$, such that $T[F, G]$ necessitates that where $x$ is $F$ there is a propensity for $\mathrm{x}$ to be $G$.

Again, $2^{\text {nd }}$ degreers will have to say something about propensities (and causes) at some stage. But there's no particular reason to think $2^{\text {nd }}$-order theorists can't do that.

\subsection{What is the basis of $N[F, G]$ 's power to constrain?}

It is now time for the crucial question: in virtue of what do $2^{\text {nd }}$-order facts like $N[F, G]$ necessitate $1^{\text {st }}$-order instantiations of natural properties, as in $\boldsymbol{M M}$. Consider $N[F, G]$. It has, given $\boldsymbol{M M}$, a necessary connection with the general fact that every $x$ that is $F$ is $G$. The connection is not analytic. The fact $N[F, G]$ is not defined in terms of the fact that every $F$ is $G$. If that were so, $N[F, G]$ couldn't metaphysically constrain the distribution of $F$ and $G$ instances, since it would be that distribution, and so the $2^{\text {nd }}$-degree view would collapse into Humeanism. What then is the connection? The fact $N[F, G]$ is a $2^{\text {nd }}$-order atomic fact involving a relation $N$, and two quiddities $F$ and $G$. The general fact, Every $F$ is $G$, is a fact concerning many first-order facts involving objects' instantiating $F$ and $G$. The fact $N[F, G]$ does not contain Every $F$ is $G$; the real definition of $N[F, G]$ has nothing do with every $F$ being $G$. So $M M$ violates $N N C$; it involves a necessary connection ungrounded in ontological dependency—see Lewis (1983) and van Fraassen (1989).

The question is how can the $2^{\text {nd }}$ degreer conceive of the necessity in $\boldsymbol{M M}$. This is where things begin to look bad for the $2^{\text {nd }}$ degree. The sorts of things $2^{\text {nd }}$ degreers might say are listed in $(a)$ to $(d)$ below. Only $(d)$ looks like a viable option:

(a) Suppose it is a brute fact that $\boldsymbol{M} \boldsymbol{M}$ holds. But then, it is just a brute fact about worlds that Every $F$ is $G$ goes with $N[F, G]$. But this is just a $2^{\text {nd }}$-order version of transworld Humeanism, one according to which facts like $\boldsymbol{M} \boldsymbol{M}$ just reflect how the worlds are. But then $N[F, G]$ would not, after all, be a source of necessitation in the world. The necessitation 
would be, on the contrary, just a brute fact about the worlds at large. $N[F, G]$ would not constrain how $F$ and $G$ are instantiated any more than $F^{1}$ and $F^{2}$ constrain $G$ 's instantiation in $\boldsymbol{M}$, according to brute-modalism. If so, the $2^{\text {nd }}$ degree to physical modality would disappear.

(b) To retain the idea that $N[F, G]$ constrains lower-level facts, $2^{\text {nd }}$-degreers might say $N$ gains its modal power by itself entering a $3^{\text {rd }}$-order fact. Suppose that $U$ is a regularity universal, viz, $U[F, G]$ obtains iff Every $F$ is $G$. Suppose then that there is a $3^{\text {rd }}$-order relation, $Z$ such that $Z[N, U]$ necessitates that if $N[F, G]$ holds then $U[F, G]$. Postulating such a relation $Z$ just transfers the problem of brute necessary connection to a higher level. In virtue of what does $Z[N, U]$ necessitate the regularity, if $N[F, G]$ holds then $U[F, G]$ ? If we answer, It is brute, then we lose $R$ 's power to constrain and thus $N$ 's power. All we have instead are brute patterns of property instantiations across worlds: brute-modality. ${ }^{14}$

(c) $2^{\text {nd }}$ degreers might hope to explain the constraining power of $N$ through a property world-making conception (Sect. 1) of modality. So, the $N$-relations are quiddities whose inherent features shape worlds, as tile-shapes determine possible tessellations. $N$ would be a kind of glue that imposes co-ordination of instantiation on $F$ and $G$. The problem with this idea is that property world-making, as we saw, just seems to require postulation of higherorder brute necessary connections. So again, we fail to explain the constraining power of $N$.

(d) Finally, the $2^{\text {nd }}$ degreer might hold that $N$ is inherently powerful, in the way that the powers theorists ( $3^{\text {rd }}$ degreers) say all natural properties are. The necessity of $\boldsymbol{M M}$ just flows from the nature of $N$. It is a kind of $2^{\text {nd }}$-order power.

I think $(d)$ is the only position left for the $2^{\text {nd }}$ degree. That means the fortunes of the $2^{\text {nd }}$ degree, ironically, depend on the fortunes of the $3^{\text {rd }}$ degree. If option $(d)$ fails, the $2^{\text {nd }}$ degree disappears. We lose the idea that the source of physical modality lies in $2^{\text {nd }}$-degree facts. ${ }^{15}$ Rather, its source is just worlds in totality, as the brute-modalist says. The $2^{\text {nd }}$ degree then depends on the $3^{\text {rd }}$ degree forming a coherent idea of properties as inherently powerful.

\footnotetext{
${ }^{14}$ See Bird 2005 for a discussion of this regress. Bird does not consider brute-modalism as an option.

${ }^{15}$ Armstrong (1993) argues that the fact $N[F, G]$ is really a fact linking states of affairs types, that is, $x$ is $F$ and $x$ is $G$. The link is causation, thought of a relation on type- rather than token-events. So $N[F, G]$ really has the form Cause $[x$ is $F, x$ is $G]$. Armstrong seems to think that we explain how $\boldsymbol{M M}$ holds hereby. How this is meant to work remains mysterious. Why would a primitive relation, cause, holding between types, necessitate that all instances of one type, $x$ is $F$ be accompanied by instances of another, $x$ is $G$ ? It is never explained.
} 


\subsection{Variations of the $2^{\text {nd }}$ degree}

Before we look at the prospects of inherently powerful properties, and saving $N$ as a constraining relation, we should look in passing at some variations within the $2^{\text {nd }}$ degree, which will be important in our evaluation of $3^{\text {rd }}$-degree theories below. Armstrong takes $N[F, G]$ to be metaphysically contingent. That is consistent with $\boldsymbol{M} \boldsymbol{M}$ holding. $\boldsymbol{M} \boldsymbol{M}$ just tells you that if $N[F, G]$ obtains it necessitates a regularity. But $\boldsymbol{M M}$ does not entail that $N[F, G]$ must obtain. Given the contingency of $N[F, G]$ we get the contingency of physical law. But $2^{\text {nd }}$ degreers do not have to affirm the contingency of law. There are two ways they can deny contingency of law and remain $2^{\text {nd }}$ degreers.

The first way is a semantic fix. Assume that $N[F, G]$ is metaphysically contingent. Think of all the $N$-relations linking all these quidditistic properties. The quiddities and their $N$-relations form a network wherein each quidditistic property has some position $Y$ in the network. Suppose then that a natural property, as we ordinarily conceive of it, is a quiddity $p$ in so far as it has network-position $Y$. The in-so-far-as-locution expresses an identity condition for an object $y$ defined by $y$ 's identity with some entity $x$ and another condition, as in:

$y$ is $x$-in-so-far-as-it-is- $F=_{\mathrm{df}} y=x \& x$ is $F$.

For example, a passenger $y$ is a person $O$ in so far as they are conveyed by some vehicle for some journey, which means that the identity conditions of the passenger $y$ is that they are identical to the person $O$ and $O$ is associated in the right way with some journey-vehicle pair. To be $x$-in-so-far-as-it-is- $F$ is not to be identical to $x$, even if being identical to $x$ is part of what being- $x$-in-so-far-as-it-is- $F$ is. It is not identity, but a condition defined in terms of identity.

Applying that idea to the term $p$ in so far as it has network-position- $Y$, the latter denotes the entity $x$ that is (i) identical to the quiddity $p$, and (ii) in the network position $Y$. The network position we shall propose requires that the same properties entering into the $N$ network relations in the actual world are present. Thus, although a quiddity $p$ might in other worlds have different network positions, or none at all, in thinking of it as a natural property of the familiar kind we are thinking of it as retaining this position in the network, and so we 
ignore those other worlds in which it has other network relations. In short, across worlds, the property $m$ is just that the property in any given world that is identical to $p$ and in network position $Y$ (along with all its quidditistic mates, from the actual world.)

On this view the laws are necessary. However, the necessity of laws does not flow in any metaphysically deep way from natures of properties, since the properties are simply defined as quiddities participating in such and such necessitations. The link between properties (quiddities) and facts of necessitation is purely notional. It is not a metaphysical link - the quiddity has no internal link to power, and the natural property is nothing but quiddity and power at those worlds where they happen to be conjoined.

The second way to get the necessity of laws is to postulate a brute necessary connection between $F$ and $G$ and $N$. That is, $N[F, G]$ is brutely necessary. Brute necessary connections are consistent with quidditism (Sect. 1). The brute necessity of $N[F, G]$ is perfectly consistent with the $2^{\text {nd }}$ degree, since all the latter requires is the non-bruteness of the connection between $N[F, G]$ and Every $F$ is $G$. So, affirming the brute necessity of $N[F, G]$ represents another variation of the $2^{\text {nd }}$ degree.

\section{Beyond the $1^{\text {st }}$ and $2^{\text {nd }}$ Degree Towards the $3^{\text {rd }}$ degree.}

The landscape of the $1^{\text {st }}$ and $2^{\text {nd }}$ degree has emerged. Here are the views we have discerned, which all accept quidditism (Quid above) about natural properties - what fixes the identity of a natural properties resides in something non-modal. However, they are linked to varying attitudes to $N N C$, the principle that there are no necessary connections ungrounded in ontological dependency, and brute-modalism:

$1^{\text {st }}$ degree:

(i) Accept combinatorialism, and thus $\mathbf{N N C}$, and embrace Humeanism.

(ii) Accept brute-modality, and deny $N N C$ for natural property instantiations, and embrace transworld Humeanism.

(iii) Accept combinatorialism, and thus $N N C$, but embrace primitive world-similarity, that is, primitive facts of comparative similarity between worlds grounding counterfactuals. 
$2^{\text {nd }}$ degree

(i) Deny $\mathbf{N N C}$ and affirm that contingent, $2^{\text {nd }}$-order-relational facts, like $N[F, G]$, constrain natural property distributions, like Every $F$ is $G$.

(ii) As in (i), but hold that natural properties are quiddities $F$ and $G$, etc, in so far as they have a position $Y$ in the network of $N$-relations.

(iii) As in (i), except affirm that the $2^{\text {nd }}$-order-relational facts $N[F, G]$ are necessary.

In the case of the $2^{\text {nd }}$ degree, we noted that even then we have variations, if instead of $N$ relations, we use $T$-relations, or even a mix thereof.

Here's the crucial issue. If there is to be a $2^{\text {nd }}$ degree at all, then $2^{\text {nd }}$-order facts like $N[F, G]$ have to constrain or govern $1^{\text {st }}$-order facts of natural property instantiation. But as we have seen, that requires that $N[F, G]$ necessitates lower order facts by virtue of the inherent nature of $N$. But this means $N$ has to be powerful in a way analogous to the $3^{\text {rd }}$ degreer's powerful natural properties. Thus, the $2^{\text {nd }}$ degree needs something like a powers view of $N$ and $T$, otherwise it collapses into brute-modalism. If the $2^{\text {nd }}$ degree affirms brute-modality for $N$, then it ceases to be a $2^{\text {nd }}$-degree view. It is just a variation on transworld Humeanism; physical modality is fixed by worlds-at-large. If the powers view proves to be an illusion, brute-modalism and quidditism are unavoidable, then, the only real views of physical modality are $1^{\text {st }}$-degree views. So let us move to the $3^{\text {rd }}$ degree to see what it is made of.

\subsection{A World of Essentially Powerful Properties?}

What $3^{\text {rd }}$ degreers promise is a vision of properties with inherent natures that in and of themselves determine property modal-roles. Properties are not merely necessarily linked to modal roles, through brute violation of $N N C$, but essentially, by their very natures, incorporating or determining modal roles or powers.

The idea, central to the $3^{\text {rd }}$ degree, that powers are inherent to properties, can be taken in two ways. The first is that properties lack any quiddity and incorporate in their essences modal role - call this the pure powers view. The second is that properties are somehow qualitative (quiddistic) but somehow produce or ground powers. Call this the powerful qualities view. Consider the pure powers view first. It may look incoherent for this reason. If 
we deny there is any quidditistic (qualitative) nature to properties, are we not affirming that there is nothing categorical to the being of properties, so how can properties be instantiated at all? There would be no concrete way-things-are since nothing would ever be actualized. This is the always-packing-never-travelling objection (Martin 1993).

This objection is confused. A property's being instantiated and its being manifested are distinct things. The always-packing objection confuses instantiation with manifestation. Think of an unmanifested dispositional property $D$. Even if $D$ is unmanifested, $D$ 's instantiation is still something in reality. What is the being of the instantiated property $D$ if it is unmanifested? The pure powers theorist should say that D's instantiation is a concrete potentiality. $D$ 's instantiation is nothing but the potentiality of another condition $C$ to be instantiated. In short, concrete reality comprises objects instantiating properties, where property instantiations are nothing but concrete potentialities, some of which are manifested, others of which are not. ${ }^{16}$

The always-packing-never-travelling objection fails. So there is no argument based thereupon that properties must have a qualitative or categorical side, as Martin (2008) and Heil (2003) urge. All the pure powers theorist need say is that property instantiations are categorical in the bland sense that they are items of being: an instantiated property is a concrete potentiality. ${ }^{17}$ How are we to understand these pure, non-quidditistic properties whose instantiations are concrete potentialities? ${ }^{18}$ I look at the three ways below:

1. The way of relational constitution: natural properties have their identities fixed by their possessing certain higher-order modal relations.

2. The way of graphs: natural properties are literally nodes in a graph, whose arc is a modal relation. The identity of properties is fixed by their contextual position in the graph.

\footnotetext{
16 This implies that when a disposition is manifested, the disposition's instantiation (by an object) is part of the resulting causal process. Causes and effects, events, are made up of concrete potentialities.

17 Strawson (2008) thinks he can establish an identity of dispositionality and categoricity in a substantial sense by simply noting: 'All being is categorical because that is what it is to be'. But this dictum just repeats the neutral thesis that a property is something that can be instantiated.

18 Psillos (2006) thinks a pure powers view generates a regress. He asks: what is the being of a property $\mathrm{P}$ when it is unmanifested on the pure powers view? Psillos replies: $\mathrm{P}$ has a power to be manifested, which is a property $\mathrm{P}_{1}$ of $\mathrm{P}$. What is this property $\mathrm{P}_{1}$ ? It must be a power, since all properties are powers. As a powerful property it must have a power to be manifested. But what's that? A property $\mathrm{P}_{2}$ ot $\mathrm{P}_{1}$. And so on. There is a quick response to this regress argument: The original property $\mathrm{P}$ just $i$ s the power, that is, the instantiation of the property is simply the potentiality of other properties to be instantiated. So the regress cannot get going.
} 
3. The way of functional roles: natural properties are constituted by functional roles. These are the three main pure powers views about properties.

On the other hand, another approach is to accept that properties, although qualitative, ground powers. This is the powerful qualities approach:

4. The way of powerful qualities: Natural properties are qualitative but their instantiations in and of themselves make modal facts, like counterfactuals, the case.

On this view, qualities do not incorporate powers as internal to their natures - like the first three ways - rather qualities somehow generate or produce modality.

These are the four $3^{\text {rd }}$-degree powers views we shall examine (as outlined in Sect 0 ). The sine qua non of a successful metaphysics of the $3^{\text {rd }}$ degree is to show either that natural property essences can include a modal dimension, or qualitative essences can, in and of themselves, ground modal facts. I argue now that our four views do not achieve this.

\section{The Way of Relational Constitution}

Our first way into the $3^{\text {rd }}$ degree is that the identities of natural properties are fixed by their entry into certain $2^{\text {nd }}$-order modal relations with respect to each other. ${ }^{19}$ What $2^{\text {nd }}$-order relations is the $3^{\text {rd }}$-degree relationalist appealing to? The relations usually cited include the stimulus-and response relation and Martin's (2008) mutual-manifestation-partner relation. Let us look at these in turn. Standardly, dispositions are characterized by their stimulus, or manifestation, conditions and their responses. Fragility is a disposition that, if instantiated, is manifested if a dropping event occurs, generating a shattering response. The three properties, fragility, being dropped, and shattering, are linked by the stimulus-response relation. This is a $2^{\text {nd }}$-order relation whose instantiation by properties explains why events featuring those properties enter into certain patterns of physical necessitation and causation. It is because the properties fragility, being dropped, and shattering bear this relation to each other, that it is metaphysically determined that if an object instantiates fragility and being dropped, it will

${ }^{19} \operatorname{Bird}(2006,2007)$ is explicit about his relationalism. See also Mumford (2004). 
instantiate the property shattering. In short, $2^{\text {nd }}$-order relations constrain first-order property instantiation. Sound familiar? This is just what $2^{\text {nd }}$ degreers say about $N$ - and $T$-relations.

Hints of the $2^{\text {nd }}$ degree also arise when we consider the mutual-manifestation-partner relation. If instantiation of fragility and being dropped by an object produces instantiation of shattering, then fragility and being dropped are partners each participating in manifesting instantiation of shattering. There is no ontological difference between the properties playing the role of stimulus, or disposition, or response, in the sense that all are powerful. At some stage, all play roles as partners which together, instantiated, can issue in manifestations, and at some stage, all play roles in which other properties, instantiated together, can produce their instantiations as outcomes. This is just what powers theorists mean when they say the powers are spread around. It is not an accidental fact that fragility and being dropped, instantiated together, produce the instantiation of shattering. It is not accidental because these properties bear the mutual-manifestation-partner relation to each other. In short, a $2^{\text {nd }}$-order relation, holding of properties, constrains how the properties are instantiated in the world — shades of the $2^{\text {nd }}$ degree again.

We see from this brief description of the stimulus-response relation and the mutualmanifestation-partner relation that both play a governing (constraining) role. That is the same role the $2^{\text {nd }}$ degreer's $N$ - and $T$-relations play. ${ }^{20}$ What then is the difference between the $2^{\text {nd }}$ degree and the $3^{\text {rd }}$-degree relationalist approach? The difference must be that the $3^{\text {rd }}$ degreer wants their $2^{\text {nd }}$-order relations to play a constituting role in fixing the identity of natural properties, so as to avoid quiddities, whereas the $2^{\text {nd }}$ degree does not. To make that clearer, consider the two roles, governing and constituting, that a $2^{\text {nd }}$-order relation, $X$, might play:

Role 1 (Governing Role): $X$ holding of $F$ and $G$ determines that if $F$ is instantiated by object $x$ then $G$ will be instantiated, (or will tend to be), by $x$ or something related to $x$. Role 2 (Constituting Role): $X$ holding of $F$ and $G$ enters into the constitution of $F$ and $G$. The identities of $F$ and $G$ are fixed by their entering into $X$-relations.

\footnotetext{
20 Schrenk (2011) and Mumford and Anjun (2011) seem to be talking about a relation holding between natural properties that looks very like a $T$-relation.
} 
The $2^{\text {nd }}$ degreer wants their $N$ - and $T$-relations to play Role 1 , the governing role, but not to play Role 2 . The $3^{\text {rd }}$ degreers wants their $2^{\text {nd }}$-order relation to play both roles.

Is it coherent to think a relation, $X$, can play both such roles? It certainly is. The governing role is to do with what constrains instantiation of properties $F$ and $G$. The constitution role is to do with what fixes the identity of the properties $F$ and $G$. We can see the two roles coming together in the following way. Think of there being a (very complex) network of $2^{\text {nd }}$-order relational facts, facts of the from $X[F, G]$. The participation of $F$ and $G$, etc, in this network determines their identity as natural properties. But, at the same time, the network determines how the participants, $F$ and $G$, are spread throughout the worlds, that is, what the patterns of their instantiation will be in the concrete world of events. ${ }^{21}$

At this point it may seem obvious that the $3^{\text {rd }}$ degreer's $X$-relations- the stimulusresponse and the mutual-manifestation-partner relation - are just the $2^{\text {nd }}$ degreers $N$ - or $T$ relations in so far as they play both governing and constituting roles. So the difference between the $2^{\text {nd }}$ degree and the $3^{\text {rd }}$-degree relationalist is simply this: the $2^{\text {nd }}$ degreer thinks the $N$ - and $T$-relations play only one role, and the relationalist thinks they play both. This is what I think is the case. But the $3^{\text {rd }}$ degreer cannot welcome this conclusion.

The worry, raised in section 2.2 , is that $N$ - and $T$-relations are just quidditistic relations that have brute necessary connections to $1^{\text {st }}$-order natural properties. If so, they have no power to constrain, and the $2^{\text {nd }}$ degree disappears as a distinct position from the $1^{\text {st }}$ degree. We can only escape this conclusion if we find some way to see $N$ - and $T$-, and thus $X$ relations as inherently powerful, in the sense that its inherent to these relations that $X[F, G]$ necessitates every $F$ is $G$, and so on. We were hoping that the powers view might help us here. We now see that the relational powers view faces the same problem. Can it escape the problem? Using its own resources, it cannot. According to relationalists, if $X$ is powerful, then that can only be because $X$ is relationally constituted through a higher-order relation $R$

\footnotetext{
${ }^{21}$ Bird is quite happy to call facts like $X[\mathrm{~F}, \mathrm{G}]$ laws - in that respect he matches Armstrong. Mumford (2004) presents the pure powers view as entailing that there are no laws. But Mumford implicitly accepts that properties entering into higher-order modal relations, like $X$-relations, fix the identities of properties. Mumford's view then differs only verbally from Bird's. Mumford reserves the term law for relations that play only a governing role, not both a governing and constituting role.
} 
that is powerful, viz, it governs $X$ 's behaviour. But clearly, we embark on a regress of how relations can govern. How does $R$ have this power, and so on?

To avoid regress, the relationalist must do one of two things. The first is to accept brute-modalism by, for example, affirming that as a matter of brute fact, the regularity, every $F$ is $G$ goes with $X[F, G]$. And so on. But then, $X$ ceases to constrain. Relationalism collapses into the $1^{\text {st }}$ degreer's brute-modalism, just as the $2^{\text {nd }}$ degree does (see Sect. 2.2). The only difference is that quiddities have structural essences — as in Role 2. But merely affirming structural quiddities is not a powers view. The structures also have to constrain natural property instantiation, but that is the issue at stake here. ${ }^{22}$

The other option is to hope for an alternative conception of the powerfulness of properties that can be applied to $X$. So relationalism depends on some (non-relationalist) way to make the $3^{\text {rd }}$ degree work. We look at other approaches below. But for now we address another question. Does the second aspect of relationalism, summed up in Role 2, work? This is the essence of the relationist approach. I want to show, now, that it utterly fails. Relational constitution of properties promises a nice image, but there is no metaphysical reality behind it. It's informative to look at relational constitution of properties since there is also a broader moral for relationalist ontologies in general.

\subsection{Relational Constitution}

First, let us be clear what relational constitution is not. The relationalist's idea that properties are constituted by their $X$-relations to other properties isn't that they are relational properties — pace Heil (2003: ch. 10). You get a relational property by taking a relational fact, like Tan is next to Jan, and removing an object, Tan, to get the property being-next-to-Jan. Or you take the relation, being-next-to, and plug a terminus with an object Jan, to get the relational property being-next-to-Jan. (By terminus I mean the unsaturated place in the relation that makes contact with $O$ when $O$ instantiates the relation.) In contrast, a natural property $F$ being constituted by its $X$-relations to other properties cannot mean that we take a relation $X$ and plug it with a material object $O$, so that $F$ is bearing- $X$-to- $O$, since $X$ is a $2^{\text {nd }}$-order

\footnotetext{
22 See Barker (2009) and Barker and Smart (2012), for related discussion.
} 
property and cannot be plugged by $O$. Nor can it mean that we take $X$ and plug it with a property, $G$, so that the property $F$ is bearing-X-to- $G$. That is because the relational property bearing- $X$-to- $G$ is a $2^{\text {nd }}$-order property, which holds of natural properties, but $F$ is meant to be a $1^{\text {st }}$-order property (holding of material objects). So what is it for $F$ to be relationally constituted?

It is meant to be this: $F$ 's identity is fixed by the unique pattern of $X$-relations it bears to other properties (Bird 2007). ${ }^{23}$ In short, $X$ is plugged by properties $F$ and $G$, (in a network of other $X$-relations) and thereby the things plugging $X$ have their natures fixed. But if this is the picture we should be very puzzled by it. Part of the essence of any property is its adicity. Its adicity is the number of places (termini) the property has, be it 1-place, 2-place, etc. The addicity of spherical is 1 : it is a 1 -place property. The adicity of being-next-to is 2 : it is a 2place relation. It is not that sphericity might have been a relation, or spatial adjacency might have been a (single-place) property. Adicity is clearly part of property essence and thus identity. So, by virtue of instantiating $X$-relations $F$ must gain some specific adicity (along with any other characteristics it has)! But how does F's being, say, a 1-place (monadic) property get to be fixed by its instantiating the higher-order relation $X$ ?

You might object that fixing identity does not require fixing adicity, even though adicity is part of the essence of a property. But what would this mean? Perhaps you think there might be identity-less things with adicity that gain identity by entry into relation with other identity-less things? That does not look coherent. Clearly, there are some issues to think about here. To do so, we need to explore a bit more what we mean be relational constitution.

\footnotetext{
${ }^{23}$ In Bird's vision of property-identity fixation, the identity (nature) of a natural property is not asymmetrically dependent on the identity of other natural properties. It is not that one natural property, derives its identity from others, which in turn rest on others. If that were the position relationalism would - as Lowe (2006) points outbe committed either to vicious circularity or infinite regress. Rather, properties altogether gain their identities by their unique network positions in the great array of $X$-relational facts in which they participate.
} 


\section{Relational Constitution Unravelled}

When philosophers theorize about how a thing might be constituted through properties or relations they typically have in mind a concrete particular $O$. Here there are two basic conceptions of how this works:

The thin or bare particular theory: $O$ is comprised by a thin particular $O^{*}$ that is thickened by its instantiation of universals;

The bundle theory: $O$ is a bundle of universals.

Now, the relationalist says that natural properties are constituted through $2^{\text {nd }}$-order relations. So, what they are proposing must be a higher-level version of one of these conceptions. Let us explore this idea. The table below sets out how we can understand this:

\begin{tabular}{|c|l|l|}
\hline & \multicolumn{1}{|c|}{ Particulars } & \multicolumn{1}{c|}{ Natural Properties } \\
\hline 1 & $\begin{array}{l}\text { Thin particulars instantiating non- } \\
\text { relational properties }\end{array}$ & $\begin{array}{l}\text { Thin properties instantiating (non- } \\
\text { relational) properties. }\end{array}$ \\
\hline 2 & $\begin{array}{l}\text { Thin particulars instantiating } \\
\text { purely relational properties. }\end{array}$ & $\begin{array}{l}\text { Thin properties instantiating purely } X- \\
\text { relations to other thin properties. }\end{array}$ \\
\hline 3 & $\begin{array}{l}\text { Bundles of non-relational and } \\
\text { relational properties. }\end{array}$ & $\begin{array}{l}\text { Bundles of non-relational and relational } \\
2^{\text {nd }} \text {-order properties. }\end{array}$ \\
\hline 4 & $\begin{array}{l}\text { Bundles of relations (relational } \\
\text { properties). }\end{array}$ & $\begin{array}{l}\text { Bundles of } X \text {-relations whose other relata } \\
\text { are } X \text {-relation bundles. }\end{array}$ \\
\hline
\end{tabular}

The left-hand column, 1-4 above, gives the basic views for concrete particulars. Standardly, people think of concrete particulars as constituted by their intrinsic properties, not by their relational properties. A chair may be constituted in part by its relations to a social context of chair producers, but typically we think its intrinsic (or non-relational) properties are primary like being chair-shaped. In short, relations must link things that are ultimately constituted independently of relations. The standard views are in left cells 1 and 3, above.

The non-standard view about particulars is that they are constituted purely relationally_left-hand cells 2 and 4-see Dipert (1997). Our chair is constituted purely by its relations to other things. Even its possession of a shape would involve a relation to something else. The right-hand column above gives the corresponding theories of 
constitution for properties. The right-hand shaded cells, 2 and 4, are counterparts for the property case of the left-hand cells 2 and 4 . The shaded cells are the two alternative accounts of natural properties thought of as constituted by $X$-relations: (i) they are thin properties thickened by their instantiation of $X$-relations to other thin properties; (ii) they are bundles of $X$-relations. Let us now go through these two theories, and see if they deliver.

\subsection{Thin and Thick Properties}

According to the first view properties are amalgams of thin properties instantiating $X$ relations. A thin property is analogous to a thin or bare particular. So let us see what a thin particular is meant to do, and then draw from that our model for thin properties. The thin particular is a kind of substratum, which, through its instantiating properties, constitutes the thick particular. The thin particular $O^{*}$ itself is not constituted by its instantiation of properties. Its role in a theory of material object constitution is to provide:

(a) the concreteness that material objects have (properties being purely abstract);

(b) the particularity that material objects have, distinguishing objects that may be qualitatively identical to each other.

We can see that both roles sit best with the standard, monadic conception of thin-particulars thickened by properties, that is, the top left cell above. Problems occur when we move to a relationalist conception of material objects, according to which an object $O$ 's identity is fixed by its entering into relations with other objects. The problem is that the thin particular $O^{*}$ brings its identity with it, even if it is a bare, colourless identity. But that is in tension with the fact that $O$ 's identity is fixed by the object's position in the network of relational facts it participates in. The latter is redundant, since $O$ gets its identity from the thin particular $O^{*}$. All the relations do is give colour to $O$, they do not determine its identity.

The concrete-object relationalist might suppose they can solve this problem by not investing any identity into the thin particular. So we deny $(b)$, and just keep $(a)$. But this idea requires introducing a concrete thing, a bare particular, without identity. What's a concrete thing without identity? Indeed, what is a plurality of concrete things, $O^{*}{ }_{1}, O^{*}$, etc, without identity? If there are a plurality of things, then we can distinguish the entities, $O^{*}{ }_{1}, O^{*}$, etc. 
But if they are distinguishable, they must have identity, since identity is linked to difference from other things, and to being countable. You cannot count things that have no identity. If so, dropping (b) is not an option. Entities require identity, and if thin particulars are entities, wholly distinct from properties and relations they have, then their identity must be independent. Having relations may colour them, but it won't determine their identity. If that is right, the material-relational constitution theorist cannot really use them.

All this would appear to show that the thin-particular approach won't work for the relationalist about material objects. The moral here carries over to the position of the relationalist about properties that invokes thin properties in its account of relational constitution. A thin property would play analogous roles to (a)-(b) above. For a natural property $F$, the thin property $F^{*}$ would:

(i) determine that $F$ was a $1^{\text {st }}$-order property instantiable by material things, with an adicity, be it monadic, two-place, three-place, etc;

(ii) provide an aspect that distinguishes $F$ from other thin properties across worlds. So, (i) is the analogue of (a) and (ii) the analogue of (b). But just as (b) caused a problem for the relationalist about material objects, (ii) causes a problem for the relationalist about natural properties. Thin-properties are meant to be completely distinct from the $X$-relations they have, but if there is a plurality of thin properties, they must have an identity. Each thin property is distinct from all others. If so they exist as entities in their own right with their own identity; the network does no job of fixing identity. But that means they are very much like quiddities. Anterior to $X$-relations, thin properties have adicity as well as identity. It looks like we have quiddities on the menu again.

Just as the material-object relationalist attempted to jettison (b), the property relationalist might attempt to dump (ii). But it won't work. The relationalists, on the thinproperty model, need a plurality of thin properties, but they can only have that plurality if thin properties possess identity and distinctness from each other. The quiddities remain. ${ }^{24}$

\footnotetext{
24 The relationalist might bit the bullet and accept thin quidditistic properties, but remind us that thin properties are thickened by their entry into $X$-relational facts. Thick properties have an essential modal aspect, even if it clothes a quidditistic core. There is an option for the relationalist, if we can make sense of thickening. But we cannot. For example, one idea is that the thickened natural property is the thin property in so far as it has that its
} 


\subsection{Bundle Theory}

The thin-property model does not provide us with a theory of relational constitution. Let us move to our second model: the bundle theory. Just as the bundle theory applied to material objects rids us of unwanted substrata - the thin particular — we might get a comparable payoff if we apply the bundle theory to properties: no property substratum or quidditistic thin-property to contend with. The image below captures the idea of a plurality of bundlesthe circles — which are natural properties, linked by $X$-relations - the arrows:

\section{Diagram 1}

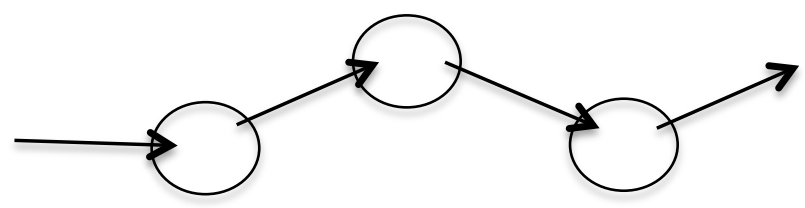

The picture is clear enough, but now we have to interpret it. What exactly are these bundles?

The standard bundle theory of material objects claims that material objects are bundles of monadic properties. But how can we envision a relational-bundle theory? The $X$ relation has order built into it. It has (at least) two places, or termini, one for the necessitator and one for the necessitated. Depending on which terminus $F$ and $G$ combine with, we get a distinct fact: $X[F, G]$ or $X[G, F]$. Thus in $X[F, G]$, the property $F$ is attached to the first terminus of $X$, and $G$ is attached to the second. If the bundle-theory of properties is to take into account $X$-relations a property cannot simply be a bundle of $X$-relations (there's only one after all). It must rather be a bundle of termini. So, if the property $F$ is a bundle of relational properties involving $X$, then if $X[F, G]$ holds, that means the first terminus of $X$ is in $F$ 's bundle. So, natural properties must be bundles of termini of X-relations. We represent each bundle like this, where little circles are the termini:

\section{Diagram 2}
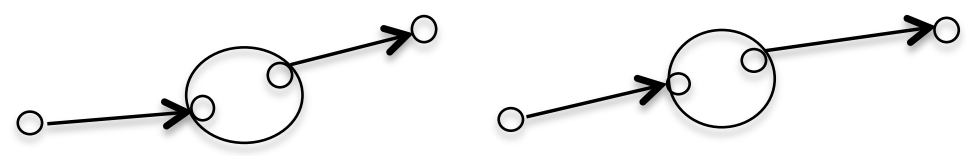

network-position in the vast network of $X$-relational facts. However, as we have already seen-Sect. 2.3- the in-so-far-as-locution introduces a mere notional connection. 
Will this provide us with the right theory of bundles? It won't.

Not all bundles bear the $X$-relation to each other: some do, some do not. (In Diagram 1, the extreme left bundle, $B 1$ is not directly linked by $X$ to the extreme right bundle, $B 3$.) The problem is that there is nothing in the structure of bundles, as so far described, that determines that a given pair of bundles, say, $B 1$ and the middle bundle, $B 2$, bear the $X$ relation to each other. Each bundle contains both termini of the $X$-relation-since all natural properties play each role: the necessitator role and the necessitated role. The fact that $B 1$ and $B 2$ contain termini of the $X$-relation cannot be what determines that they bear the $X$-relation to each other, for if it did, every bundle would bear $X$ to every other. We need to add another ontological ingredient to the bundles to get the fact of relation that $B I$ bears the $X$-relation to $B 2$. Here a number of equivalent ideas suggest themselves, but all lead to the collapse of the bundle theory. Here's one.

We postulate a plurality of quidditistic $X$-relations, $X^{1}, X^{2}$, etc, each qualitatively distinct from the other. Between each bundle, there is a distinct relation $X^{\mathrm{i}}$ with its distinct termini. Diagram 3 pictures this, where $X^{1}, X^{2}$, etc are depicted by arrows with different kinds of broken lines, indicating distinct $X$-relations:

\section{Diagram 3}

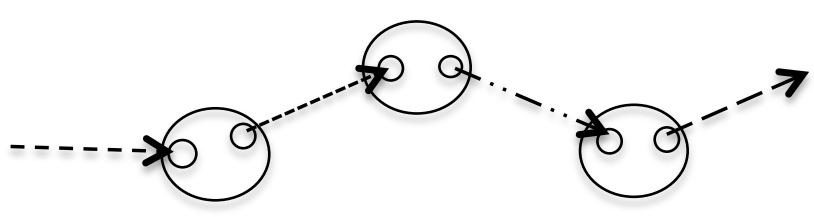

$X^{1}$ links $B 1$ and $B 2$ because one of its termini is in each bundle. That is what the fact of relation consists in. And so on for other the relations $X^{2}, X^{3}$, and other bundles.

The problem now is how do all these quiddistically distinct $X$-relations get linked to the same constraining role? Assuming quiddities can generate roles at all, we need some quiddistic essence they all have in common. But that is what we are now denying. The 
bundle theory faces a dilemma: it needs a unique $X$-relation to determine a governing role, but it needs a diversity of $X$-relations to get the relational connectedness of bundles. ${ }^{25}$

An objection at this point is that if the relational bundle theory for properties fails, why does it not fail for material objects? That is a good question, but not an objection to the argument just given. The thesis that material objects are constituted by bundles of (asymmetric) $1^{\text {st }}$-order relations is in trouble as well. So ontic structural realism-see Ladyman and Ross (2007) — is problematic if it embraces relational bundle theory.

Leaving aside this problem of relating bundles, the issue of adicity remains. Suppose natural properties differ on their adicity: some are 1-place properties, like charge, others 2-or more place relations, like spatial relations. On the bundle theory, all natural properties are just bundles of $X$-relation termini. So how do differences of adicity arise from bundling? The number of $X$-relation termini in any given bundle cannot be the determiner, since the number of termini just reflects the adicity of the $X$-relation. If all bundles are linked by a 2-place $X$ relation, all bundles have two termini-see Diagram 1. Suppose bundle $B 1$ is a $1^{\text {st }}$-order relation, whereas $B 3$ is not a relation. Where does $B 1$ 's being a relation come from? Nothing in the structure of bundles determines that fact. One can't just add termini to bundles as a kind of outer layer, since termini are not separable entities that can be added to something. We cannot, for example, add a thin property with adicity to the bundles. For how is the thin property glued to the bundle? ${ }^{26}$ I conclude: the bundle theory can't explain adicity, the most basic feature of the identity of a property.

\subsection{Property Priority-Monism}

We have made no progress with either thin properties or bundles as views about what relational constitution of properties resides in. ${ }^{27}$ Here's a new tack, which uses Schaffer's

\footnotetext{
${ }^{25}$ One might say these are $X$-relation instances, but just one $X$-relation. But instances of relations are, apparently, ontologically dependent on their relata. But then, natural properties depend on $X$-property instances, but the latter depend on natural properties. The analysis would then be circular.

${ }^{26}$ This problem is essentially the same problem, which we considered above, of how thin properties (with adicities) are thickened. We are just facing it from the other side, as it were.

27 One might analyse property instantiation in terms of partial identity (Baxter 2001). Applied to relations, this approach faces essentially the same problem as the bundle theory. Another idea is that properties are abstractions from prior states of affairs. This does not sit well with a $3^{\text {rd }}$ degree approach, for if objects' having properties are primary, where properties are just abstractions therefrom, it's unclear how higher-order facts $X[F, G]$ can constrain lower level facts, since the former are just abstractions from the latter.
} 
(2010) priority monism. A relationalist might embrace a priority-monism for properties, according to which all properties depend for their being on one basic thing: the network of properties. For Schaffer, a plurality of things are all dependent on a whole by virtue of their being linked by an internal constraining relation. For Schaffer, a relation $R$ is internal in this sense just in case $R$ 's holding of $A$ and $B$ determines that how, where, or whether $A$ is places a constraint on how, where, or whether $B$ is (2010: p. 352). On this definition the $X$-relation is an internal relation, since by virtue of holding of $F$ and $G$, how, where, or whether $F$ is places a constraint on how, where, or whether $G$ is. That is, $F$ 's being instantiated necessitates $G$ 's being instantiated. So, by Schaffer's definition, the $X$-relation is internal, and so, by Schaffer's criteria, property priority-monism holds.

The problem is that Schaffer's conception of internal relation is wrong. On his definition, Armstrong's necessitation relation, $N$, is internal, since its holding of $F$ and $G$ constrains how these properties are instantiated. But $N$ is not an internal relation as such. In Armstrong's system, $N$ has nothing to do with the nature of $F$ and $G$, for it is a contingent matter that $N$ holds of $F$ and $G$. Schaffer is mistaking a merely necessary relation with an internal (essential) relation. He does this because his discussion tacitly assumes there cannot be necessary connections ungrounded in ontological dependency, that is, he assumes combinatorialism about possibility. For a combinatorialist, if $A$ 's bearing $R$ to $B$ constrains how things are with $A$ and $B$, it is because $A$ 's bearing $\mathrm{R}$ to $B$ is part of $A$ 's real definition, or vice versa. That is why Schaffer thinks constraining relations constitute the internality of the relation. But once we see beyond combinatorialism, we see this idea of internal relation is utterly flawed. ${ }^{28}$

I conclude, that at best the $3^{\text {rd }}$-degree relationalist view fails to provide any way in which modal role is internal to the essence of properties, even assuming we can solve the problem of how the $2^{\text {nd }}$-order $X$-relation governs. There's no access to the $3^{\text {rd }}$ degree here.

\footnotetext{
${ }^{28}$ Can the relationalist just treat essentiality as primitive, and affirm that natural properties are thin properties instantiating $X$-relations essentially, and not merely necessarily, and leave it at that? But if the essences of thin properties are non-modal, what would their essentially instantiating $X$-relations amount to? It is utterly unclear.
} 


\section{The Way of Graphs}

The way of the relationalist approach to the $3^{\text {rd }}$ degree is a dead end for the powers theorist. We must look elsewhere for a $3^{\text {rd }}$-degree theory of properties. Here we turn to our second way to the powers view, the graph-theoretic way (Sect. 3.1). This way moves up a level of abstraction with the hypothesis that natural properties are nodes in an abstract relational structure, called a graph. Graphs have been receiving a good deal of discussion with respect to holistic ontologies. Some theorists, as we shall see, think that nodes in graphs are purely relationally constituted beings. So, graphs might give us a way of understanding properties as relationally constituted beings that avoids the fatal objections we have just uncovered. (In fact, I think this is false.) Or perhaps graphs give us a different path altogether. To see if these hopes can be realised, we need a brief introduction to graphs and their metaphysical natures.

In what follows, I shall distinguish between graph-diagrams, diagrams of various kinds on paper or computer screens, and graphs proper, the supposed abstract structures they represent. Graphs are mathematical structures with two kinds of kinds of entities, nodes and arcs. The nodes and arcs of graphs are represented in graph-diagrams:

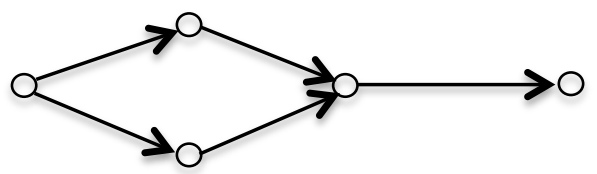

Circles are nodes, and arcs arrows. Diagrams can be labelled and unlabelled. Non-labelling means that the represented nodes cannot be distinguished outside of the structure of the graph. The lines of graph-diagrams can have arrows attached to them, as ours above does. This represents the fact that the arc, which corresponds to a relation or relation type, is nonsymmetric.

The graph-diagram, a collection of lines and circles, is a representation of a graph. The graph is a kind of abstract entity. But what kind is it? I will argue that a graph is the relational structure of an ensemble of things bearing relations to each other. 
Suppose that in the graph-diagram above the arrows represent causation. The graph then represented by the diagram is a structure realized by different groups of five events in relations of causation. The nodes in the graph are not themselves events. Rather, they are types of events. For example, the far left node of this 5-cause graph is the type of entity whose tokens cause two other events that in turn cause (independently) another event, which causes a fifth event. And so on. The graph-diagram above can represent many such graphs for particular asymmetric relations. But it can also represent an inherently more general graph: one of a greater degree of abstraction. This is the graph that is the relational structure of five things bearing any asymmetric relation to each other in the pattern displayed. In this graph, call it the 5-asymmetric-relation graph, the arc is not a relation, but a relation-type whose tokens are specific asymmetric relations like cause or being-older-than, and so on. The nodes are entity-types relationally defined: types whose tokens are entities in a group of five objects whose members bear some asymmetric relation $R$ to each other in the pattern displayed by the graph-diagram.

We can see the same idea of graphs - as the relational structures of ensembles of things bearing relations to each other-within the philosophy of mathematics in the pattern structuralism of Resnik (1997) and Shapiro (1997). For the pattern structuralist, number theory is about the structure common to all $\omega$-sequences. For example, the two sequences of sets below, Zermelo's and von Neuman's respectively, are particular $\omega$-sequences:

0

1

2

3 $\varnothing$

$\{\varnothing\}$

$\{\{\varnothing\}, \varnothing\}$

$\{\{\{\varnothing\}, \varnothing\},\{\varnothing\}, \varnothing\}$

etc. $\varnothing$

$\{\varnothing\}$

$\{\{\varnothing\}\}$

$\{\{\{\varnothing\}\}\}$

etc.

According to the structuralist, we cannot identify the sequence of natural numbers with either sequence of sets. The numbers are not sets, rather they are nodes in a graph with an infinite set of nodes whose arc is the successor relation, as in:

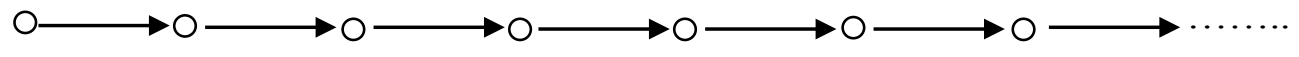


The numbers are types whose tokens are members of $\omega$-sequences. The successor relation is itself a relation type. Instances of the successor relation are asymmetric, non-transitive relations, for example, the set-theoretic relations that hold between the sets in the set $\omega$ sequences instanced above. Thus, the number-graph is like the 5-asymmetric relation graph in having an arc that is a relation type - a type with relations as its tokens - and not a relation as such.

If we are to make use of graphs in our theory of natural properties - using the hypothesis that natural properties are nodes in some kind of graph — we need to know what nodes are. Nodes seem to have no interior, no non-relational nature. As Shapiro (1997) states, they are structureless atoms or points in a structure. That might sound promising for an antiquidditisic theory of properties. Shapiro's enticing suggestion might be taken as the view that nodes are purely relationally constituted things: things whose only properties are relational. If so, arcs are relations instantiated by the nodes of the graph. Certainly, theorists like Dipert (1997) and Ladyman (2007) seem to have this view. However, the idea that nodes are relationally constituted entities in this sense is completely wrong. Nodes cannot be entities that instantiate their arcs - thought of as relations - for the following reason.

The arc of the 5-cause graph is the relation of causation, but the nodes are not things instantiating that relation. After all, the nodes, qua abstract entities, are not causing each other. That would be a gross confusion. The 5-cause graph is the structure realized by five concrete events when some of these concrete events cause each other, as indicated. It is the realizing concrete events that instantiate cause, not the nodes that are components of the structure that is realized. In the 5-asymmetric relation graph, the nodes cannot be instantiating the arc, since the arc is not even a relation; it is a relation type, a pure structure. Likewise, in the number graph, the nodes cannot be instantiating the arc, since, according to the structuralist, the arc of this graph is a relation type whose tokens include certain settheoretic relations, such as those holding between the sets we displayed above. Therefore, as a relation type its not the sort of thing that can be instantiated.

The relational constitution idea about nodes is completely off track. A better theory of nodes lies with what we have been suggesting all along. They are types. In particular, they 
are what we might call relational abstractions: they are types that identify tokens purely by appeal to relations, (or types of relations) those tokens bear to each other. In the 5-cause graph, the nodes are types whose tokens are entities, events, instantiating causal relations. In the 5-asymmetric-relation graph, the nodes are types whose tokens are members bearing some asymmetric relation $R$ to each other. In the number graph the nodes, the numbers, are types whose tokens are particular members of $\omega$-sequences. Relational abstractions-types that identify particulars purely by appeal to relations - are entities whose identity is given by the purely relational features of their tokens.

I will not explore here how we should understand types qua abstract entities. I shall just assume, as seems right, that they are identified by their actual or potential tokens. There is no demand that the tokens of the type exist, just as ante rem structuralism (Shapiro 1997) does not require that structures be realized by anything in order to exist.

How do these results bear upon the project of a powers theorist aiming to explain the weaving of modal pattern into properties using graphs? Well, the idea is that natural properties are nodes in some graph. So the obvious thought is that the graph concerned is one whose arcs are the $X$-relation (or family of such relations, that is, the stimulus-response relations or mutual-manifestation partner relations). Allowing for extreme simplification, think of the first graph-diagram as representing the graph for the natural properties, where the arc is the $X$-relation. Call this the $X$-graph. Will this get us a theory of natural properties? No. The problem is that the nodes in the $X$-graph are no more properties than the nodes of the 5cause graph are events. Rather the nodes will be property types. Let us see why.

As I proposed above, graphs are relational abstractions: the nodes are types of entities that instantiate relations to each other. If the arc of the $X$-graph is the $X$-relation then the nodes are types, whose tokens (if they exist) are properties instantiating $X$-relations to each other. So the nodes are not themselves natural properties, but rather natural-property types. If the nodes are property types in this sense, they are not properties, and cannot be instantiated by material objects, what gets instantiated are their tokens. Analogy: the type colour-property is not a property instantiated by any object; it is not a property. If that is right, we do not have a theory of properties. We have a theory of something else: property types. 
If we want natural properties to be nodes in a graph, the relation, or arc, of the graph cannot be $X$. (Otherwise we just get the $X$-relation graph.) The graph's relation must be some other relation $R$, relevant to physical modality, holding of things other than properties. So here is an idea. The $3^{\text {rd }}$ degreer might claim that $R$ is causation, a relation holding of eventtokens. So, the natural property graph is just like the 5-cause graph-its arc is causationbut, of course, it is a much more complex graph. Call this the Z-cause graph. Graph nodes, as we have observed, are types. So, if the arc for the Z-cause graph is causation, a relation linking event-tokens, then properties, on the present hypothesis, are event-types whose tokens are particular events in causal relations to other particular events.

The idea then is that natural properties are event-types. They are relational abstractions, in the sense defined above, whose tokens are events in causal relations. An object $O$ instantiates a natural property $F$ if and only if the token event $[O$ is $F$ ] is a token of an event-type that is a node of the $Z$-cause graph. $[O$ is $F]$ can be a token of a node of the $Z$ cause graph if and only if $[O$ is $F]$ is in a pattern of events, linked by causation, with the same structure as the $Z$-cause graph. The pattern of causation must realize the structure of the $Z$ cause graph. So, if $O$ has natural property $F$, the $Z$-cause graph has to be realized in the world @. Now for the crucial question. What is the Z-cause graph, such that it is realized in @? The Z-cause graph is such that its realizer is either @'s total causal history or some sub-history of @. Both views conflict with our pre-theoretic commitments about natural properties.

Suppose it is the whole history of events in @ that is the realiser set of the Z-cause graph. Then each node of the graph is only realized once, since the graph is just the structure of the network of actual causal unfolding of unique events. But then, given properties are nodes, that is, event types, each property is only instantiated once. But properties are multiply instantiated in our world. So, the realizer set of the Z-cause graph can't be the total history of @. It must be a sub-history of @. Suppose, a set of events, $e^{1}, e^{2}$, etc, is a proper part of the causal history of@ and that this is the realizer of the Z-cause graph. The token events $e^{1}, e^{2}$, etc are events that involve instantiation of properties, $F^{1}, F^{2}$, etc. These properties have to be instantiated outside of the realizer set, since we want each natural property, $F^{\mathrm{i}}$ to be multiply instantiated. Therefore, token-events $f$ outside the realizer set have to be tokens of the same 
types as token-events $e^{\mathrm{i}}$ inside the realizer set. But what's the basis of the type identity? The identity fixed by the graph is relational: two token-events are the same type just in case they enter into the same causal relations. But $f$ outside the realizer set is not in the same causal relations as $e^{\mathrm{i}}$ inside. If so, they cannot be of the same type. The only way out of this is to suppose that the world is a mosaic of perfectly resembling sub-histories each being a perfect realizer of the $Z$-cause graph. We have no reason to suppose this is right.

To conclude: if the $X$-relation is the arc of a graph — the $X$-graph— the nodes are the wrong type-level to be natural properties. If cause is the relation of the graph-the $Z$-cause graph - the nodes have the wrong identity conditions to be natural properties. I see no other alternatives for the graph-theoretic approach. So, graphs won’t deliver natural properties.

\section{The Way of Functional Roles}

We now come to the third way in which the $3^{\text {rd }}$ degree might be developed. It is the idea that the powerfulness of properties resides in their being constituted by functional roles.

Functional roles can be understood through patterns of counterfactuals. Armstrong (1997, p.79), somewhat metaphorically, characterizes this possibility when he says that the powers theorist thinks of properties as 'congealed hypothetical facts or states of affairs'. We might put the thought this way: an object $O$ 's instantiation of a natural property is just for the object to be part of a pattern of counterfactual facts concerning how $O$ and related objects behave under hypothetical circumstances. ${ }^{29}$

The counterfactuals that enter into the constitution of natural properties, according to the functional-role theory, are counterfactuals that map out the stimulus and response conditions that are linked to any property. So, suppose a given property $F$ has stimulus condition $G$ and manifestation condition $H$. (My ' $F$ 's, ' $G$ 's, and ' $H$ 's are ambiguous between property names and predicate letters.) Let '>' be short for ' _ were the case, _ would be the case'. Then we can say:

\footnotetext{
29 This conception of properties as congealed counterfactual facts is found in Holton (1999), and defended against Blackburn (1990). However, it may have its origins in Shoemaker (1980). See also Hawthorne's (2001) causal structuralism. Ellis and Lierse (1996) propose this kind of theory for some fundamental properties. For a more recent theory see Whittle $(2008,2009)$.
} 
$\boldsymbol{F R}: O$ has property $F$ in virtue of the fact that $(O$ is $G)>(\mathrm{O}$ is $H) .{ }^{30}$

To understand the functionalist approach, we have to understand how exactly property natures are fixed through such counterfactuals. First, let me indicate what we should not say. We should not think of functional roles as theories, which the realizing properties make true. We do not want to say this because theories are linguistic-semantic entities that describe reality and are not part of the constitution of material reality. That is to confuse the metaphysical issue with a semantic issue. So the truth-making role should not be part of the characterization of the powerfulness of properties.

This tendency to confuse metaphysical issues about the constitution of properties with semantic issues is rife. ${ }^{31}$ The use of Ramsey sentences illustrates the confusion. Take the totality of conditionals that express all the stimulus-response relations that there are. Replace all the predicate letters by variables, then add quantifiers, as below-here $p_{1}, p_{2}, p_{3}, \ldots$ range over properties, and $x_{1}, x_{2}, \ldots$ range over physical things. The result is a Ramsey-sentence:

$$
\exists p_{1} \exists p_{2} \exists p_{3} \ldots, \forall x_{1} \forall x_{2} \forall x_{3} \ldots\left(x_{1} \text { has } p_{1} \ldots>x_{1} \text { has } p_{3}\right) \& \ldots, \text { etc. }
$$

Natural properties are those properties jointly satisfying the open-sentence with existential quantifiers removed. But satisfying such an open-sentence is not what the natural properties consist in. It does not fix their identity in the metaphysical sense we are concerned with. It just provides a conceptual system enabling thinkers to determinately think about the properties in terms of the relations, or roles, that are meant to constitute them. In short, the Ramsey-sentence may provide a description of the essences of the properties, but satisfying the open-sentence isn't what their essences reside in.

We cannot say then that realizing a role resides in satisfying open Ramsey-sentences. Rather, we need something in non-linguistic/non-semantic reality for objects to participate in when they realize functional roles. I think what we need are counterfactual facts or states of

\footnotetext{
30 One might object that finks and antidotes will undermine such conditionals. Whittle (2009) argues plausibly that for fundamental (sparse) natural properties finks and antidotes either won't be present or can be neutralized by building more conditions into the antecedents of the conditionals. I remain neutral on this issue here.

${ }^{31}$ Some cases are: Hawthorne (2001), Heil (2003), Whittle (2008), and Jacobs (2011).
} 
affairs, qua non-semantic entities. What's a counterfactual state of affairs? The standard view is that counterfactual propositions are about relations between possible worlds. If so, a counterfactual fact/state of affairs is a condition about relations of comparative similarity between the actual world and other possible worlds. Such a condition is the thing in reality in the required sense to be part of the constitution of natural properties.

Counterfactual facts can be part of the constitution of natural properties in the following way. The fact that an object $O$ has a property $F$ resides in the fact the nearest $(O$ is $G$ )-worlds $w$ to the actual are ( $O$ is $H$ )-worlds, and so on. Naturally, on this conception, the fact that $O$ is $G$ at worlds $w$ is in turn constituted by a range of facts about comparative similarity relations between worlds, such as that the nearest ( $O$ is $J)$-worlds to $w$ are $(O$ is $D)$ worlds, for other natural properties, $J$ and $D$, and so on. In short, there must be a network of worlds in relations of comparative similarity involving every natural property. (That looks like what Holton (1999) proposes.)

What determines these facts of comparative similarity across worlds? It is fairly obvious that we have to say that these facts of world comparative-similarity are basic. They can't be, for example, the result of a mosaic of particular matters of fact in each world. If we thought that we would simply be reproducing Lewis's conception, the $1^{\text {st }}$-degree Humean view (Sect. 3), according to which prior facts about the mosaic of property instantiations fix world comparative-similarity.

Likewise, the functional view must also be distinct from the $1^{\text {st }}$-degree view (iii), summed up in section 3. According to the latter, each world is a mosaic of particular matters of fact, and the world comparative similarity relations are brute facts about worlds. On this view, the facts about property mosaics in particular worlds and the brute facts of similarity between worlds then determine counterfactual facts, such as that the nearest ( $O$ is $G$ )-worlds to $@$ are $(O$ is $H)$-worlds.

Both $1^{\text {st }}$-degree views, $(i)$ and (iii), affirm quidditism about properties since properties are ontologically prior to the facts of world comparative-similarity and thus to counterfactual facts. If the functionalist is to avoid sinking into quidditism, they must deny that there is a mosaic of facts in a world that is ontologically prior to facts of world comparative-similarity. 
They must say the mosaic of particular facts at a world $w$ is constituted by, posterior to, the inter-world facts of comparative similarity. After all, that is what the functionalist affirms in saying: the fact that $O$ has $F$ is constituted by the nearest ( $O$ is $G$ )-worlds being ( $O$ is $H$ )worlds, and so on. That implies that if we look at $w$ in itself, abstracted from its relations to other worlds, we cannot find any particular matters of fact in $w$. If we could discern them, then it would have to be that $w$ 's properties and their instantiations had their identities fixed independently of the relations to other worlds, which would be quidditism.

In making this claim we are not denying that there are properties at worlds. The issue is not, Are there properties instantiated at world w?, but rather What is it for properties to be instantiated at $w$ ?. The functionalist answer is: It is for $w$ to be in relations of similarity with other worlds. In brief, the fixation of properties at $w$ is an extrinsic relational matter, not an intrinsic matter. So now we can ask: What's the intrinsic nature of $w$ prior to facts of interworld similarity that determine its mosaic of particular facts?

The world $w$ must contain objects, but they cannot be objects in the full-blooded sense, thick particulars, with their properties (since properties have not been fixed yet). They must be bare particulars. Intrinsically, then $w$ is a totality of bare particulars. This is deeply problematic. If the particulars are concrete, then presumably they have spatiotemporal relations. They are regions of spacetime, or things at such regions. But spacetime is not a bare particular devoid of physical nature. It is a highly structured being whose nature must, already incorporate all the features of physical modality we expect of spacetime. This idea of bare particulars and spacetime is as objectionable as quidditism for the powers theorist. For in it, the intrinsic substance of the world lies behind physical nature, which is more or less what powers theorists want to escape from. The functionalist path is clearly failing to deliver.

Even if we could live with this conception of worlds, prior to property fixation, as structures with bare particulars, how exactly do we get properties back to clothe these worlds? The problem is that comparative similarity for counterfactuals is defined in terms of properties, as follows: the nearest $(O$ is $G$ )-worlds are ( $O$ is $H)$-worlds, etc. So, it seems, the facts of comparative similarity required by counterfactuals presuppose the isolation of properties, $G$ and $H$, etc., so cannot be used to define property identity. Look at the 
metaphysical space of worlds, each, intrinsically, without natural properties, in relations of comparative similarity. This space is just bare-particular worlds in comparative similarity relations. We can see $O$ and its counterparts across worlds, but what makes some set of worlds $(O$ is $G)$-worlds, etc? To carve out such worlds we need another ontological ingredient. I do not think we have it. ${ }^{32}$ The functionalist view is visibly failing.

Perhaps the functionalist's problem is assumption of a possible-worlds conception of counterfactuals. The alternative, a metalinguistic conception of counterfactuals, won't help, since it presupposes natural laws_-see Barker (2011). Primitivism about counterfactual facts won't help either. Suppose, all the counterfactual facts about $O$ form a compound fact:

$(O$ is $G)>(O$ is $H),(O$ is $H)>(O$ is $K)$, etc.

One might argue that participating in such a compound fact determines the identity of each property. ${ }^{33}$ This boils down to a relational-constitution conception of natural properties. The relations are counterfactual-based, for example, the relation that a property $x$ bears to $y$ when, $(O$ is $x)>(O$ is $y)$, and so on. The question now is how would this improve on the relationalconstitution approaches we have already examined and dismissed (Sect. 4-5)? I think there is essentially no difference. Moreover, there is another problem waiting in the wings.

If we are primitivists about counterfactuals, how do we understand the fact that a counterfactual's antecedent condition necessitates its consequent condition? As in:

[O is $G]$ and $(O$ is $G)>(O$ is $H)$ necessitate $[O$ is $H]$.

What's the basis of this necessity? A non-primitivist treatment of counterfactuals will explain it. But counterfactual primitivism must treat it as primitive. Looks like brute-modalism has crept in again. But that is inimical to the powers view.

The functionalist fails to deliver a view of properties has inherently powerful. We have to look elsewhere for the $3^{\text {rd }}$ degree.

\footnotetext{
32 Whittle (2008) suggests that tropes can fix the counterfactuals. So for her, in each world, there are already tropes, prior to counterfactual facts. This is equivalent to the view that worlds are mosaics of particular matters of fact, qua tropes. But that amounts to affirming that there are objective similarities at each world, prior to inter-world relations of similarity. But, by virtue of that very fact, we have embraced quidditism. Whittle's view, as we shall see in a moment, can also be interpreted as an entirely distinct theory from of the functionalrole view, namely, the powerful qualities view. We should not confuse these two approaches.

${ }^{33}$ We might think of this structure as a physical world correlate to a Ramsey-sentence.
} 


\section{The Way of Powerful Qualities}

The fourth and final way from our list of paths to the $3^{\text {rd }}$ degree (Sect. 3.1) is an impure powers view. It is the powerful qualities theory. Call it $P Q . P Q$-theorists claim that the natural properties $F$ are quiddities_-either simple or structured. Nevertheless, an object $O$ 's possessing $F$, along with the natures of other properties $G$ and $H$ make a counterfactual $(O$ is $G)>(O$ is $H)$ the case. ${ }^{34}$ In short, the set of entities comprising the fact $[O$ is $F]$ and $G$ and $H$ ground the counterfactual-below arrow is the grounding relation:

$$
\text { PQ1: }\{[O \text { is } F], G, H\} \longrightarrow(O \text { is } G)>(O \text { is } H) \text {. }
$$

We assume entities — whether facts or properties — can enter into grounding relations.

Grounding is an asymmetric determination, or ontological dependency. It is expressed by locutions like $A$ in virtue of $B, B$ makes $A$ the case. And so on. We can think of it as a kind of atemporal causation, through levels of reality rather than through time. $\mathbf{P Q 1}$ implies that the set $\{[O$ is $F], G, H\}$ in and of itself makes the counterfactual the case without the aid of laws. Laws are posterior to counterfactuals according to this approach.

To illustrate: suppose object $O$ is spherical. Then according to $P Q$, by virtue of the quidditistic nature of sphericity, given other properties, the counterfactual holds: were $O$ solid and placed on slope $S$ in a gravitational field, $O$ would role down $S$. For $P Q$, sphericity's nature is not fixed by powers - as in the relational-constitution approach — but the powers flow from or have their source in its qualitative nature. Qualities are powerful, not by virtue of being constituted by modal relations, or counterfactual structures, incorporating the power internally, but by generating or grounding modal power.

A more general way of putting $P Q$ is to propose that the totality of all quidditistic properties grounds a vast counterfactual fact involving all the properties:

\footnotetext{
34 See Heil (2003), who draws on the work of Martin (2008). See also Tugby (2012), Jacobs (2011), and also Whittle (2009). (Whittle is a trope-theorist. Tugby is open on trope theory. These theorists put their claims in terms of making counterfactuals true. I have already argued that we do not want our theory of physical reality to be couched in terms of a relation between physical facts and propositions. Instead, we should talk of counterfactual facts, the worldly correlates of true counterfactual propositions.
} 


$$
\begin{gathered}
\text { PQ2: }\left[\forall x_{1}, \forall x_{2}, \ldots\left(\left(x_{1} \text { is } F^{1}\right)>\left(\left(x_{1} \text { is } G^{1}\right)>\left(x_{1} \text { is } H^{1}\right)\right) \& \ldots(\ldots, \text { etc. })\right]\right. \\
\left\{F^{1} \ldots, G^{1} \ldots, H^{1}, \ldots, Q^{1} \ldots, \text { etc. }\right\}
\end{gathered}
$$

A fragment of this huge grounding fact, for some arbitrary object $O$, looks like this:

$$
\text { PQ3: }\{F, G, H\} \quad \longrightarrow(O \text { is } F)>((O \text { is } G)>(O \text { is } H))
$$

So, the properties, $\{F, G, H\}$, ground the counterfactual: were $O$ to be $F$, then, were it to be $G$, it would be H. (Each property is picked out in at last one clause of the corresponding counterfactual sentence. We do not need a state of affairs to play a grounding role, as in $\boldsymbol{P Q 1}$, because we have put all the possible conditions into the counterfactual.) So properties, quiddities, do the work of ground. What they ground are counterfactuals.

That is $P Q$ in brief. How are we meant to understand $P Q$ 's central claim of grounding? Let us assume that grounding implies necessity. So, in $\mathbf{P Q 3},\{F, G, H\}$ necessitates the counterfactual. This does not mean $\{F, G, H\}$ contains the counterfactual fact. The latter is not part of the real definition of $\{F, G, H\}$. The counterfactual is a distinct existence from $\{F, G, H\} .{ }^{35}$ So $\boldsymbol{P Q} 3$ violates $\boldsymbol{N N C}$. (More generally, $\boldsymbol{P Q 1 - 3}$ violate $\boldsymbol{N N C}$.) The pressing question is why isn't $P Q$ just a form of brute-modalism? The $P Q$-theorist might offer several lines of explanation for why it isn't. Following them will help us understand $P Q$ better, but also show, precisely, where it fails.

(a) The $P Q$-theorist might claim that $P Q 3$ does not in fact exemplify a brute necessary connection, ungrounded in ontological dependency, since the counterfactual is a free ontological lunch. That means it is no increase in being over $\{F, G, H\}$. This line of response by the $P Q$-theorist is dubious to say the least. The counterfactual state of affairs exists. It is either included in the real definition of $\{F, G, H\}$, or it is distinct from it, and so is an increase in being. The counterfactual fact is not part of $\{F, G, H\}$. A counterfactual is not just a set of properties. So it cannot be contained by $\{F, G, H\}$. In which case, it is an increase in being.

\footnotetext{
35 Note that this holds even if we propose that the grounding base is: $\{F, G, H,>\}$, that is, we include the form of the counterfactual state of affairs in the grounding base. I won't do that in the main text.
} 
(b) The $P Q$-theorist might claim that if $A$ grounds $B$, then $B$ is part of $A$ 's essence. So, $P Q 3$ isn't a necessary connection violating $N N C$. But this line of argument is dubious. That $A$ grounds $B$ does not imply that $A$ contains $B$. For example, assume there are disjunctive facts. Then, the existence of the fact [snow is white] grounds an infinite number of disjunctive facts of the form [snow is white $\vee Q$ ]. But the infinite number of disjunctive facts are not part of [snow is white]'s essence. The latter does not contain the disjunctive facts. So, just because something grounds a counterfactual, it does not follow that the counterfactual is part of its essence.

(c) A related idea is that it is part of $\{F, G, H\}$ 's essence to ground the counterfactual. But this idea is not the $P Q$-theory. $P Q$ says properties are quiddities that ground counterfactuals. What's now being suggested is that $\{F, G, H\}$ includes grounding- $a$ counterfactual as part of its essence. This idea is an alternative conception of powersinstanced by the first three ways to the $3^{\text {rd }}$ degree-which says properties incorporate modal role in their essences. Somehow the real definition of $\{F, G, H\}$ includes the grounding relational property of making $(O$ is $F)>((O$ is $G)>(O$ is $H))$ the case. Again, this is not $P Q$, but some other theory. Moreover, we have found no way so far to incorporate modality into essence. Let us then leave this view aside, and return to $P Q$.

(d) $P Q$-theorists may object that their view is not simply that $\{F, G, H\}$ necessitates the counterfactual. It is that it grounds it. Grounding implies necessitation. But the converse does not hold. The grounding relation involves an asymmetric determination, whose epistemic correlate is explanation. If $A$ grounds $B$, then $A$ explains $B$. If so, in $P Q 3,\{F, G$, $H$ \} explains the counterfactual. So, it is not a brute necessary connection, due to this explanatory connection. It is a nice idea. But is there anything to it?

The obvious concern is how this explanatory connection arises. Why does it hold between a cluster of quiddities, $\{F, G, H\}$, and a counterfactual? The explanatory connection must either be a derived fact, something explicable by the relata involved, or a brute explanatory connection. Neither option works for $P Q$. Let us see why.

For $P Q$, the explanatory connection cannot be derived. For example, one way of explaining how $\boldsymbol{P Q 3}$ holds is through a theory of counterfactuals. Unfortunately, no known 
plausible view of counterfactuals, possible worlds approaches or metalinguistic approaches, will deliver $\boldsymbol{P Q 3}$. The metalinguistic theory requires prior laws and the world-similarity approach requires prior facts of similarity, and neither is present in $\{F, G, H\}$. If we do somehow include laws or facts of world-similarity, we end up with a different view about physical modality, and not $P Q . P Q$ says $\{F, G, H\}$ by itself makes the counterfactual the case - not with the help of laws and similarity relations.

Another false hope of explaining the explanatory connection in $\mathbf{P Q 3}$ is to bring in the property world-making conception of real possibility (Sect. 1). Property world-making was the idea that the inherent qualitative natures of properties shape worlds they are instantiated in, just as tile-shape constrains tessellations. But, as we saw, this idea just invokes primitive brute-modalism. So, this last option for the $P Q$-theorist fails. The explanatory connection in $P Q 3$ cannot be a derived fact.

The $P Q$-theorist must say then that it is just a brute fact that $\{F, G, H\}$ grounds, and thus explains, the counterfactual. (The counterfactuality must itself be primitivecounterfactuals cannot be explained by laws or world-similarity for $P Q$, as we have seen.) The problem now is that we cannot simply stipulate that one kind of thing explains another. Suppose that $F$ is some physical property and $G=$ pain-qualia. The primitive pain-qualia is utterly distinct in nature from the physical property. What sense can we make of the thesis that $O$ 's having $F$, given pain-qualia's primitive nature $G$, grounds $O$ 's being in pain:

$$
\{[O \text { is } F], G\} \quad \rightarrow \quad[O \text { is } G] .
$$

How could the obtaining of $F$ explain the obtaining of $G$ ? It can't. So we can't simply affirm it as a hypothesis. What stands in the way is the explanatory gap. If qualia facts are primitive (constitutionally) their obtaining cannot be explained by physical facts. If you can provide a theory of qualia states that closes the gap — say they are functional states realizable in physical states - you have an explanation. That requires that the qualia state is not completely distinct from the physical state. Otherwise, all you have is psycho-physical dualism, in which, as a matter of brute necessity, certain qualia just happen to go with certain physical properties. The $P Q$-theorist, on the present understanding, is in exactly this position. 
The only way for the $P Q$-theorist to truly affirm $P Q 3$ is to close the explanatory gap between quiddity and modal fact. To do that they need a plausible, non-primitivist theory of counterfactuals, or a property-world-making theory of modality. They have neither. I conclude then that the powerful properties theory cannot distinguish its claims $P Q 1-3$ from affirmations of brute modality. If so, brute-modalism is back and $P Q$ is a mirage.

\section{Twilight of The Powers}

We have looked at relational constitution, graphs, and functional roles, and powerful qualities as ways of articulating the idea of inherently powerful properties. Each of these proposals has failed to produce the result wanted by the $3^{\text {rd }}$ degreer: a metaphysics of properties as sources of physical modality. Where do we go from here?

The $3^{\text {rd }}$-degree theorist might be tempted by the idea that what's gone wrong is our choice of relations, like the stimulus-response relation, as the physical aspects upon which to build the $3^{\text {rd }}$ degree. They might look to something else: forces or fields. But this won't provide a better view of properties. It seems natural to say that forces/fields are just dispositional properties of spatiotemporal regions. Their dispositionality just brings us back to stimulus-response relations, whose limitations we have already revealed.

Another idea is that we involve functional laws, which concern determinable quantities rather than determinate properties-like $F=M A$. A functional law, like $F=M A$, is the fact that all the determinants of $F, M$, and $A$ form an infinite set of ordered triples, $<f, m, a>$ whose magnitudes conform to product law: the magnitude of $f=$ the product of those of $m$ and $a$. If this is what the functional law is, then it cannot constrain these determinants since it is reduced to a very complex fact involving them.

Other options for the $3^{\text {rd }}$ degree look hopeless. Molnar's (2003) physical intentionality - dispositionality is like psychological intentionality - is deeply obscure. (It is criticised elsewhere-Mumford (1999) and Whittle (2003)). Martin's (2008) identity viewalso Heil's (2003) - that categoricity just is dispositionality looks incoherent, since it implies that what fixes $F$ 's identity is non-modal, (qualitative) but also modal (dispositional). A 
double-aspect theory requires that we fuse dispositionality with quiddity as two aspects of one whole. However, our reflections should show now that anything more than a notional connection will be unachievable. Merely affirming that natural property $F$ is quiddity $q$ in so far as its has some modal role will simply get us a theory of notional connections between quiddities and facts about brute modal connection like the $2^{\text {nd }}$-degree view of section 2 .

To conclude: it looks from this survey that there is no coherent metaphysical theory of the $3^{\text {rd }}$ degree. No metaphysical theory of properties has shown how properties - be they $1^{\text {st }}, 2^{\text {nd }}, 3^{\text {rd }}$, order, monadic or relational - can incorporate modal role as part of their essences, or ground, in and of themselves, modal facts. If that is right, there is no $2^{\text {nd }}$-degree metaphysics either, since the $2^{\text {nd }}$ degree depends on certain $2^{\text {nd }}$-order relations like, $N$ and $T$, being inherently constraining, like $2^{\text {nd }}$-order powers (Sect. 2.2). The only approach left standing is the $1^{\text {st }}$ degree. Here there are the three positions we outlined below-Humeanism, transworld Humeanism, and primitive-world-similarity — and their variants. These views accept property quidditism, and either embrace combinatorialism or brute-modalism about real possibility. Thus, the actual world as a whole, or possible worlds as a totality, are the only candidates for the role of being sources of physical modality.

You might object that we cannot square this conclusion with the strong intuition that there are non-brutal necessary connections between distinct existences. Examples abound: determinants necessitating determinables, the necessitation of singleton sets by their members, or truth-making. To take the first, case, $O$ 's being scarlet necessitates its being red. One might think this is a necessity that $(i)$ is not brute, but arises out of the nature of scarlet and red, and (ii) is not merely the necessity of essence, viz, the result of scarlet's being defined in part through red. If so, surely there must be some way to understand necessary connections that are not grounded in ontological dependency (essence) that goes beyond mere affirmation of brute-modalism, since $\boldsymbol{C} \boldsymbol{L}$, scarlet's necessitating red, is an instance thereof? This objection has no force until a metaphysical theory is produced that explains the necessity of determinants and determinables. It is really doing nothing more than reminding us that the mystery of necessary connections between distinct existences goes beyond the cases presented to us by physical modality. Solving the problem is another matter. And 
indeed, it may be that these intuitions we have of non-brute necessary connections between distinct existences are simply illusions.

$1^{\text {st }}$-degree views are holistic in one sense. The world, or worlds, in totality ground modal facts. But it is not a holistic, relational ontology of the kind described by Dipert (1997) and Schaffer (2010). This paper, if anything, is negative about the prospects of holistic ontology in the sense that object identity is fixed by some prior whole, of a structural or network (see Sect. 5 and 6). Those results spill over into the $1^{\text {st }}$-order claims for what ontic structural realists and priority monists claim. I won't pursue these matters here. ${ }^{36}$

\section{References}

Armstrong, David 1983: What is a Law of Nature?, Cambridge: Cambridge University Press. 1989: A Combinatorial Theory of Possibility, Cambridge: Cambridge

University Press.

1993: 'The Identification Problem and the Inference Problem' Philosophy and Phenomenological Research, 53, pp. 421-32.

1997. A World of States of Affairs. Cambridge: Cambridge University Press.

Barker, Stephen 2009: 'Dispositional Monism, Quiddities, and Relational Constitution' Analysis, 69, pp. 242-250.

2011: 'Can counterfactuals really be about possible worlds?' Noûs, 45, pp. 1-22.

Barker, Stephen and Ben Smart 2012: 'The Ultimate Argument against Dispositional Monist Theories of Law', Analysis, 72, pp. 714-22.

Baxter, Donald 2001: 'Instantiation as Partial Identity' Australian Journal of Philosophy, 79, pp. 449-64.

Bird, Alexander 2005: 'The Ultimate Argument against Armstrong's Contingent Necessitation View of Laws' Analysis, 65, pp.147-55. 2006: ‘The Regress of Pure Powers?' Philosophical Quarterly, 57, pp. 513-34.

\footnotetext{
${ }^{36}$ I would like to thank the following for useful comments on this paper: referees from Mind, the Editor of Mind, and Toby Handfield. I also thanks: Rani Anjum, David Armstrong, Angelo Cei, Michael Esfeld, Phil Dowe, Frank Hofmann, Siegfried Jaag, Stephen Mumford, Philip Percival, Ben Smart, Matt Tugby.
} 
2007: Nature's Metaphysics, Oxford: Oxford University Press.

Black, Robert 2000: ‘Against Quidditism’ Australian Journal of Philosophy, 78, pp. 87-104.

Blackburn, Simon 1990: 'Filling in Space' Analysis, 50, pp. 62-65.

Carroll, John 1994: Laws of Nature, Cambridge: Cambridge University Press.

Dipert, Randall 1997: 'The Mathematical Structure of the World: The world as Graph', Journal of Philosophy, 94, pp. 329-358.

Dretske, Fred 1977: 'Laws of Nature' Philosophy of Science, 44, pp. 248-68.

Ellis, Brian 2001: Scientific Essentialism, Cambridge: Cambridge University Press.

Ellis, Brian and Carolyn Lierse 1994: 'Dispositional Essentialism' Australian Journal of

Philosophy: 72, pp. 27-45.

Fine, Kit 1994: 'Essence and Modality: The Philosophical Perspectives Lecture'

Philosophical Perspectives, 8, pp. 1-16.

Fraasen, Bas. van 1989: Laws and Symmetries, Oxford: Oxford University Press.

Handfield, Toby. 2008. 'Humean Dispositionalism' Australasian Journal of Philosophy, 81, pp. 113-116.

Hawthorne, John 2001: ‘Causal Structuralism’ Philosophical Perspectives, 15, pp. 361-78.

Heil, John 2003 From an Ontological Point of View, Oxford: Oxford University Press.

Holton, Richard 1999: 'Dispositions All the Way Round' Analysis, 59, pp. 9-14.

Jacobs, Jonathan 2011: 'Powerful Qualities, not Pure Powers' The Monist, 94, pp. 81-102.

Ladyman, James 2007: 'On the Identity and Diversity of Objects in a Structure' Proceedings of the Aristotelian Society LXXXI, pp. 23-42.

Ladyman, James, and Don Ross 2007: Everything must Go: Metaphysics Naturalized,

Oxford: Oxford University Press.

Lange, Marc 2009: Laws and Lawmakers, New York: Oxford University Press.

Lewis, David 1979: 'Counterfactual dependence and Time's Arrow' (plus postscripts), in

Lewis 1986, pp. 32-66. Originally published in 1979 in Nous 13.

1986: A Plurality of Worlds, Oxford: Oxford University Press.

Lowe, Edward 2006: The Four-Category Ontology: A Metaphysical Foundation for Natural Science, Oxford: Oxford University Press. 
Martin, Charles 1993: 'Power for Realists', in Ontology, Causality and Mind. John Bacon, Keith Campbell and Lloyd Reinhardt (eds.). Cambridge University Press, pp. 175-94. 2008: The Mind in Nature, Oxford: Oxford University Press.

Molnar, George 2003: Powers, Oxford: Oxford University Press.

Mumford, Stephen 1999: 'Intentionality and the Physical: A New theory of Disposition Ascription', Philosophical Quarterly, 49, pp. 215-25. 2004: Laws in Nature, London: Routledge.

Mumford, S. and Rani Anjum 2011: Getting Causation from Powers, Oxford University Press.

Psillos, Stathis 2006: 'What do powers do when they are not manifested' Philosophy and Phenomenological Research, 72, pp. 137-56.

Resnik, Michael 1997: Mathematics as a Science of Patterns, Oxford: Oxford University Press.

Schaffer, Jonathan 2009: 'Spacetime the One Substance' Philosophical Studies 145, pp. 13148. 2010: 'The Internal Relatedness of all Things' Mind, 119, pp. 341-74.

Shapiro, Stewart 1997: Philosophy of Mathematics: Structure and Ontology, New York: Oxford University Press.

Shoemaker, Sydney 1980: 'Causality and Properties' in P. van Inwagen, ed., Time and Caus, pp. 109-135.

Schrenk, Marcus 2010: 'The Powerlessness of Necessity', Noûs 44, pp. 725-739.

Strawson, Galen 2008: 'The Identity of the Categorical and the Dispositional' Analysis, 68, pp. 271-82.

Tooley, Michael 1977: 'The Nature of Laws' Canadian Journal of Philosophy, 7, pp. 667-98. Tugby, Matt 2012: 'Rescuing Dispositionalism from the Ultimate Problem: Reply to Barker and Smart'. Analysis, 72, pp. 723-31.

Whittle, Ann 2003: Review of Powers: A Study in Metaphysics by George Molnar. Human Nature Review, 3, pp. 399-401. 
2008: 'A Functionalist Theory of Properties' Philosophy and Phenomenological Research, 77, pp. 59-82.

2009: 'Causal Nominalism', in Toby Handfield (ed) Dispositions and Causes, Oxford: Oxford University Press.

Wilson, Jessica 2010: 'What's Hume's Dictum, and Why Believe it?'. Philosophy and Phenomenlogical Research, 80, pp. 595-637.

2012: 'Hume's Dictum and Metaphysical Modality: Lewis's Combinatorialism', in Barry Loewer and Jonathan Schaffer (eds) The Philosophy of David Lewis, London: Blackwell. 